\title{
Primitive ideals and automorphisms of quantum matrices
}

\author{
S Launois and T H Lenagan *
}

\begin{abstract}
Let $q$ be a nonzero complex number that is not a root of unity. We give a criterion for $\langle 0\rangle$ to be a primitive ideal of the algebra $\mathcal{O}_{q}\left(M_{m, n}\right)$ of quantum matrices. Next, we describe all height one primes of $\mathcal{O}_{q}\left(M_{m, n}\right)$; these two problems are actually interlinked since it turns out that $\langle 0\rangle$ is a primitive ideal of $\mathcal{O}_{q}\left(M_{m, n}\right)$ whenever $\mathcal{O}_{q}\left(M_{m, n}\right)$ has only finitely many height one primes. Finally, we compute the automorphism group of $\mathcal{O}_{q}\left(M_{m, n}\right)$ in the case where $m \neq n$. In order to do this, we first study the action of this group on the prime spectrum of $\mathcal{O}_{q}\left(M_{m, n}\right)$. Then, by using the preferred basis of $\mathcal{O}_{q}\left(M_{m, n}\right)$ and PBW bases, we prove that the automorphism group of $\mathcal{O}_{q}\left(M_{m, n}\right)$ is isomorphic to the torus $\left(\mathbb{C}^{*}\right)^{m+n-1}$ when $m \neq n$ and $(m, n) \neq(1,3),(3,1)$.
\end{abstract}

2000 Mathematics subject classification: 16W35, 16W20, 20G42, 81R50.

Key words: Quantum matrices, quantum minors, prime ideals, primitive ideals, automorphisms.

\section{Introduction}

The automorphism group of a polynomial algebra $\mathbb{C}\left[X_{1}, \ldots, X_{n}\right]$, for $n \geq 2$, is in general far from being understood. In the case $n=2$ the group was described explicitly by Jung, 12. However, it is only recently that Shestakov and Umirbaev, [19, have proved that the wellknown Nagata automorphism of $\mathbb{C}\left[X_{1}, X_{2}, X_{3}\right]$ is wild; that is, the Nagata automorphism cannot be written as a product of elementary automorphisms.

${ }^{*}$ This research was supported by a Marie Curie Intra-European Fellowship within the $6^{\text {th }}$ European Community Framework Programme and by Leverhulme Research Interchange Grant F/00158/X 
In this paper, we are interested in the quantum case. More precisely, when $q$ is a nonzero complex number that is not a root of unity, we study the automorphism group of the algebra $\mathcal{O}_{q}\left(M_{m, n}\right)$ of $m \times n$ quantum matrices which in turn is a non-commutative deformation of a polynomial ring in $m \times n$ indeterminates. We denote by $Y_{i, \alpha},(i, \alpha) \in \llbracket 1, m \rrbracket \times \llbracket 1, n \rrbracket$, the canonical generators of $\mathcal{O}_{q}\left(M_{m, n}\right)$. It is well-known that the group $\mathcal{H}:=\left(\mathbb{C}^{*}\right)^{m+n}$ acts on $\mathcal{O}_{q}\left(M_{m, n}\right)$ by $\mathbb{C}$-automorphisms via:

$$
\left(a_{1}, \ldots, a_{m} ; b_{1}, \ldots, b_{n}\right) . Y_{i, \alpha}=a_{i} b_{\alpha} Y_{i, \alpha} \quad((i, \alpha) \in \llbracket 1, m \rrbracket \times \llbracket 1, n \rrbracket) .
$$

(Note that this is not a faithful action; for example, $(a, \ldots, a ; 1, \ldots, 1)$ and $(1, \ldots, 1 ; a, \ldots, a)$ have the same action on $\mathcal{O}_{q}\left(M_{m, n}\right)$, multiplying each $Y_{i, \alpha}$ by $a$. This explains why the automorphism group is an $(m+n-1)$-torus rather than an $(m+n)$-torus.)

It was observed by Alev and Chamarie, [1], that quantization implies rigidity and so puts limits on the automorphism group of quantized algebras. This explains why it has been possible to compute the automorphism group of several quantum algebras at least in the generic case. For instance, Alev and Chamarie, [1], have described the automorphism group of the algebra of $2 \times 2$ quantum matrices, Alev and Dumas, [2], the automorphism group of the positive part of the quantized enveloping algebra of a complex simple Lie algebra of type $A_{2}$, the first author, [13], the automorphism group of the positive part of the quantized enveloping algebra of a complex simple Lie algebra of type $B_{2}, \ldots$.

One method to study the automorphism group of an algebra is to use the invariance of the set of height one primes under the action of the automorphism group. This method was used successfully by Rigal, [18], to compute the automorphism group of quantized Weyl algebras and next by Gomez-Torrecillas and El Kaoutit, [6], to calculate the automorphism group of the coordinate ring of quantum symplectic spaces. We also use this method in the present paper. However, in the cases of quantized Weyl algebras and coordinate ring of quantum symplectic spaces, the number of height one primes is finite (because of the choice of parameters) and so the restrictions on the automorphisms are very strong. In the case of the algebra of quantum matrices, the set of height one primes is in general not finite; so the situation is substantially more complicated.

We start by considering when the algebra $\mathcal{O}_{q}\left(M_{m, n}\right)$ has only finitely many height one primes. It turns out that, because of the stratification theorem of Goodearl and Letzter, see [3] for example, this situation arises exactly when $\langle 0\rangle$ is a primitive ideal of $\mathcal{O}_{q}\left(M_{m, n}\right)$. Thus, the first section of this paper is devoted to this question: we establish a criterion for the ideal $\langle 0\rangle$ to be primitive in $\mathcal{O}_{q}\left(M_{m, n}\right)$. More precisely, we prove that $\langle 0\rangle$ is a primitive ideal of $\mathcal{O}_{q}\left(M_{m, n}\right)$ if and only if $v_{2}(m) \neq v_{2}(n)$, where $v_{2}(k)$ denotes the 2-adic valuation of a positive integer $k$. This shows, for example, that $\langle 0\rangle$ is a primitive ideal of $\mathcal{O}_{q}\left(M_{2,3}\right)$. 
This criterion together with the stratification theorem of Goodearl and Letzter shows that, if $v_{2}(m) \neq v_{2}(n)$, then $\mathcal{O}_{q}\left(M_{m, n}\right)$ has only finitely many height one primes and it turns out that they are all $\mathcal{H}$-invariant. On the other hand, if $v_{2}(m)=v_{2}(n)$, then $\mathcal{O}_{q}\left(M_{m, n}\right)$ has infinitely many height one primes. A finite number (those that are $\mathcal{H}$-invariant) are already known from previous work of the authors and Rigal, [14. In the second part of this paper, we provide an explicit generator for every height one prime of $\mathcal{O}_{q}\left(M_{m, n}\right)$. (Note that, since $\mathcal{O}_{q}\left(M_{m, n}\right)$ is a Noetherian (non-commutative) UFD, every height one prime of $\mathcal{O}_{q}\left(M_{m, n}\right)$ is generated by a normal element, [14.) Hence, in this second part, we describe all normal elements of $\mathcal{O}_{q}\left(M_{m, n}\right)$ that generate a prime ideal.

Finally, in the third section, we investigate the automorphism group of $\mathcal{O}_{q}\left(M_{m, n}\right)$ when $m \neq n$. Using the description of the height one primes of $\mathcal{O}_{q}\left(M_{m, n}\right)$ together with graded arguments, we show that all height one primes that are $\mathcal{H}$-invariant, except possibly one, are invariant under every automorphism of $\mathcal{O}_{q}\left(M_{m, n}\right)$. Next, by using the preferred basis of $\mathcal{O}_{q}\left(M_{m, n}\right)$ and certain PBW bases, we are able to prove that the automorphism group of $\mathcal{O}_{q}\left(M_{m, n}\right)$ is isomorphic to the torus $\left(\mathbb{C}^{*}\right)^{m+n-1}$ when $m \neq n$ and $(m, n) \neq(1,3),(3,1)$. This latter restriction occurs because of the existence of a rogue automorphism for quantum 3space (which may be viewed as $1 \times 3$ quantum matrices), see [1]. This is the only exception, and our analysis recovers the Alev-Chamarie result for this exceptional case.

In the case where $m=n$, the algebra $O_{q}\left(M_{n}\right)$ has a homogeneous central element of degree $n$, the quantum determinant. The existence of this element considerably complicates the computation of the automorphism group, since the graded arguments that we use in the non-squared case do not put strong limits on the automorphism group and its action on the height one primes in the square case. In addition, transposition provides an automorphism that also clouds the analysis. The conjecture is that the automorphim group of $\mathcal{O}_{q}\left(M_{n}\right)$ is generated by a torus and the transposition automorphism. This has been verified by Alev and Chamarie, [1, in the $2 \times 2$ case. Our present methods can recover the Alev-Chamarie result, but, as yet, we are unable to deal with the general case, although we do have partial results. We intend to return to this question in a subsequent paper.

\section{A criterion for quantum matrices to be primitive.}

In this section, we use the $\mathcal{H}$-stratification theory of Goodearl and Letzter together with the deleting derivations theory of Cauchon in order to characterize the integers $m$ and $n$ such that $\langle 0\rangle$ is a primitive ideal in $\mathcal{O}_{q}\left(M_{m, n}\right)$. 


\subsection{The $\mathcal{H}$-stratification of the prime spectrum of $\mathcal{O}_{q}\left(M_{m, n}\right)$.}

Throughout this paper, we use the following conventions.

- If $I$ is a finite set, $|I|$ denotes its cardinality.

- $\llbracket a, b \rrbracket:=\{i \in \mathbb{N} \mid a \leq i \leq b\}$.

- $\mathbb{C}$ denotes the field of complex numbers and we set $\mathbb{C}^{*}:=\mathbb{C} \backslash\{0\}$.

- $q \in \mathbb{C}^{*}$ is not a root of unity.

- $m, n$ denote positive integers.

- $R=\mathcal{O}_{q}\left(M_{m, n}\right)$ is the quantization of the ring of regular functions on $m \times n$ matrices with entries in $\mathbb{C}$; it is the $\mathbb{C}$-algebra generated by the $m \times n$ indeterminates $Y_{i, \alpha}, 1 \leq i \leq m$ and $1 \leq \alpha \leq n$, subject to the following relations:

$$
\begin{aligned}
Y_{i, \beta} Y_{i, \alpha} & =q^{-1} Y_{i, \alpha} Y_{i, \beta}, & & (\alpha<\beta) ; \\
Y_{j, \alpha} Y_{i, \alpha} & =q^{-1} Y_{i, \alpha} Y_{j, \alpha}, & & (i<j) ; \\
Y_{j, \beta} Y_{i, \alpha} & =Y_{i, \alpha} Y_{j, \beta}, & & (i<j, \alpha>\beta) ; \\
Y_{j, \beta} Y_{i, \alpha} & =Y_{i, \alpha} Y_{j, \beta}-\left(q-q^{-1}\right) Y_{i, \beta} Y_{j, \alpha}, & & (i<j, \alpha<\beta) .
\end{aligned}
$$

It is well-known that $R$ can be presented as an iterated Ore extension over $\mathbb{C}$, with the generators $Y_{i, \alpha}$ adjoined in lexicographic order. Thus the ring $R$ is a Noetherian domain; we denote by $F$ its skew-field of fractions. Moreover, since $q$ is not a root of unity, it follows from [10, Theorem 3.2] that all prime ideals of $R$ are completely prime. We denote by $\operatorname{Spec}(R)$ the set of (completely) prime ideals of $R$.

- It is well-known that the algebras $\mathcal{O}_{q}\left(M_{m, n}\right)$ and $\mathcal{O}_{q}\left(M_{n, m}\right)$ are isomorphic. Hence, we assume that $m \leq n$.

- It is easy to check that the group $\mathcal{H}:=\left(\mathbb{C}^{*}\right)^{m+n}$ acts on $R$ by $\mathbb{C}$-algebra automorphisms via:

$$
\left(a_{1}, \ldots, a_{m}, b_{1}, \ldots, b_{n}\right) . Y_{i, \alpha}=a_{i} b_{\alpha} Y_{i, \alpha} \quad \text { for all } \quad(i, \alpha) \in \llbracket 1, m \rrbracket \times \llbracket 1, n \rrbracket .
$$

An $\mathcal{H}$-eigenvector $x$ of $R$ is a nonzero element $x \in R$ such that $h . x \in \mathbb{C}^{*} x$ for each $h \in \mathcal{H}$. An ideal $I$ of $R$ is said to be $\mathcal{H}$-invariant if $h . I=I$ for all $h \in \mathcal{H}$. We denote by $\mathcal{H}$-Spec $(R)$ the set of $\mathcal{H}$-invariant prime ideals of $R$. Since $q$ is not a root of unity, it follows from [9, $5.7]$ that $\mathcal{H}$-Spec $(R)$ is a finite set. Note that $\langle 0\rangle$ is an $\mathcal{H}$-invariant prime ideal of $R$, since $R$ is a domain.

The action of $\mathcal{H}$ on $R$ allows us to use the $\mathcal{H}$-stratification theory of Goodearl and Letzter, see [3, II.2], to constuct a partition of $\operatorname{Spec}(R)$ as follows. If $J$ is an $\mathcal{H}$-invariant 
prime ideal of $R$, we denote by $\operatorname{Spec}_{J}(R)$ the $\mathcal{H}$-stratum of $\operatorname{Spec}(R)$ associated to $J$. Recall that $\operatorname{Spec}_{J}(R):=\left\{P \in \operatorname{Spec}(R) \mid \bigcap_{h \in \mathcal{H}} h . P=J\right\}$. Then the $\mathcal{H}$-strata $\operatorname{Spec}_{J}(R)$, with $J \in \mathcal{H}$-Spec $(R)$, form a partition of $\operatorname{Spec}(R)$, see [3]:

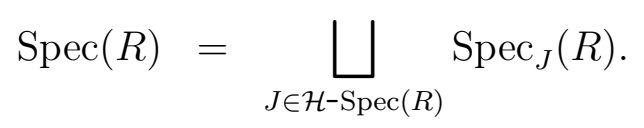

Naturally, this partition induces a partition of the set $\operatorname{Prim}(R)$ of all (left) primitive ideals of $R$ as follows. For all $J \in \mathcal{H}$-Spec$(R)$, we set $\operatorname{Prim}_{J}(R):=\operatorname{Spec}_{J}(R) \cap \operatorname{Prim}(R)$. Then it is obvious that the $\mathcal{H}$-strata $\operatorname{Prim}_{J}(R)(J \in \mathcal{H}$-Spec $(R))$ form a partition of $\operatorname{Prim}(R)$ :

$$
\operatorname{Prim}(R)=\bigsqcup_{J \in \mathcal{H}-\operatorname{Spec}(R)} \operatorname{Prim}_{J}(R) .
$$

One of the reasons that makes the $\mathcal{H}$-stratification interesting is that it provides a powerful tool for recognizing primitive ideals. Indeed, since $\mathbb{C}$ is uncountable and since the Noetherian domain $R$ is generated as an algebra by a finite number of elements, it follows from $[3$, Proposition II.7.16] that the algebra $R$ satisfies the Nullstellensatz over $\mathbb{C}$, see [3, II.7.14]. Further the set of $\mathcal{H}$-invariant prime ideals of $R$ is finite. Thus [3, Theorem II.8.4] implies that $\operatorname{Prim}_{J}(R)(J \in \mathcal{H}$-Spec$(R))$ coincides with the set of those primes in $\operatorname{Spec}_{J}(R)$ that are maximal in $\operatorname{Spec}_{J}(R)$. Hence, we deduce the following criterion for $R$ to be a primitive ring.

Proposition $1.1 R$ is a primitive ring if and only if the $\langle 0\rangle$-stratum of $\operatorname{Spec}(R)$ is reduced to $\langle 0\rangle$; that is, $\langle 0\rangle$ is the only prime ideal in $\operatorname{Spec}_{\langle 0\rangle}(R)$.

\subsection{Dimension of the $\langle 0\rangle$-stratum of $\operatorname{Spec}(R)$.}

In this section, we show that the $\langle 0\rangle$-stratum $\operatorname{Spec}_{\langle 0\rangle}\left(\mathcal{O}_{q}\left(M_{m, n}\right)\right)$ is Zariski-homeomorphic to the prime spectrum of a commutative Laurent polynomial ring in $\operatorname{dim}(\operatorname{ker}(B))$ indeterminates, where $B$ is a $m n \times m n$ matrix with entries in $\mathbb{C}$. First, we describe the matrix $B$ explicitly and then we will compute the dimension of the kernel of $B$.

First recall, see [5], that the theory of deleting derivations can be applied to the iterated Ore extension $R=\mathbb{C}\left[Y_{1,1}\right] \ldots\left[Y_{m, n} ; \sigma_{m, n}, \delta_{m, n}\right]$ (where the indices are increasing for the lexicographic order $\leq$ ). The corresponding deleting derivations algorithm is called the standard deleting derivations algorithm. Before recalling its construction, we need to introduce some notation. 
- We denote by $\leq_{s}$ the lexicographic ordering on $\mathbb{N}^{2}$. We often call it the standard ordering on $\mathbb{N}^{2}$. Recall that $(i, \alpha) \leq_{s}(j, \beta)$ if and only if $[(i<j)$ or $(i=j$ and $\alpha \leq$ $\beta)]$.

- We set $E_{s}=(\llbracket 1, m \rrbracket \times \llbracket 1, n \rrbracket \cup\{(m, n+1)\}) \backslash\{(1,1)\}$.

- Let $(j, \beta) \in E_{s}$. If $(j, \beta) \neq(m, n+1)$, then $(j, \beta)^{+}$denotes the least element (relative to $\left.\leq_{s}\right)$ of the set $\left\{(i, \alpha) \in E_{s} \mid(j, \beta)<_{s}(i, \alpha)\right\}$.

As described in [5], the standard deleting derivations algorithm constructs, for each $r \in E_{s}$, a family $\left(Y_{i, \alpha}^{(r)}\right)_{(i, \alpha) \in[1, m] \times[1, n]}$ of elements of $F:=\operatorname{Frac}(R)$, defined as follows.

1. If $r=(m, n+1)$, then $Y_{i, \alpha}^{(m, n+1)}=Y_{i, \alpha}$ for all $(i, \alpha) \in \llbracket 1, m \rrbracket \times \llbracket 1, n \rrbracket$.

2. Assume that $r=(j, \beta)<_{s}(m, n+1)$ and that the $Y_{i, \alpha}^{\left(r^{+}\right)}((i, \alpha) \in \llbracket 1, m \rrbracket \times \llbracket 1, n \rrbracket)$ are already constructed. Then, it follows from [4, Théorème 3.2.1] that $Y_{j, \beta}^{\left(r^{+}\right)} \neq 0$ and, for all $(i, \alpha) \in \llbracket 1, m \rrbracket \times \llbracket 1, n \rrbracket$, we have:

$$
Y_{i, \alpha}^{(r)}= \begin{cases}Y_{i, \alpha}^{\left(r^{+}\right)}-Y_{i, \beta}^{\left(r^{+}\right)}\left(Y_{j, \beta}^{\left(r^{+}\right)}\right)^{-1} Y_{j, \alpha}^{\left(r^{+}\right)} & \text {if } i<j \text { and } \alpha<\beta \\ Y_{i, \alpha}^{\left(r^{+}\right)} & \text {otherwise. }\end{cases}
$$

As in [4], we denote by $\bar{R}$ the subalgebra of $\operatorname{Frac}(R)$ generated by the indeterminates obtained at the end of this algorithm, that is, we denote by $\bar{R}$ the subalgebra of $\operatorname{Frac}(R)$ generated by the $T_{i, \alpha}:=Y_{i, \alpha}^{(1,2)}$ for each $(i, \alpha) \in \llbracket 1, m \rrbracket \times \llbracket 1, n \rrbracket$.

Let $N \in \mathbb{N}^{*}$ and let $\Lambda=\left(\Lambda_{i, j}\right)$ be a multiplicatively antisymmetric $N \times N$ matrix over $\mathbb{C}^{*}$; that is, $\Lambda_{i, i}=1$ and $\Lambda_{j, i}=\Lambda_{i, j}^{-1}$ for all $i, j \in \llbracket 1, N \rrbracket$. We denote by $\mathbb{C}_{\Lambda}\left[T_{1}, \ldots, T_{N}\right]$ the corresponding quantum affine space; that is, the $\mathbb{C}$-algebra generated by the $N$ indeterminates $T_{1}, \ldots, T_{N}$ subject to the relations $T_{i} T_{j}=\Lambda_{i, j} T_{j} T_{i}$ for all $i, j \in \llbracket 1, N \rrbracket$. In [5, Section $2.2]$, Cauchon has shown that $\bar{R}$ can be viewed as the quantum affine space generated by the indeterminates $T_{i, \alpha}$ for $(i, \alpha) \in \llbracket 1, n \rrbracket^{2}$, subject to the following relations.

$$
\begin{aligned}
& T_{i, \beta} T_{i, \alpha}=q^{-1} T_{i, \alpha} T_{i, \beta}, \quad(\alpha<\beta) ; \\
& T_{j, \alpha} T_{i, \alpha}=q^{-1} T_{i, \alpha} T_{j, \alpha}, \quad(i<j) \text {; } \\
& T_{j, \beta} T_{i, \alpha}=T_{i, \alpha} T_{j, \beta}, \quad(i<j, \alpha>\beta) ; \\
& T_{j, \beta} T_{i, \alpha}=T_{i, \alpha} T_{j, \beta}, \quad(i<j, \alpha<\beta) .
\end{aligned}
$$


Hence $\bar{R}=\mathbb{C}_{\Lambda}\left[T_{1,1}, T_{1,2}, \ldots, T_{m, n}\right]$, where $\Lambda$ denotes the $m n \times m n$ matrix defined as follows. We set

$$
A:=\left(\begin{array}{ccccc}
0 & 1 & 1 & \ldots & 1 \\
-1 & 0 & 1 & \ldots & 1 \\
\vdots & \ddots & \ddots & \ddots & \vdots \\
-1 & \ldots & -1 & 0 & 1 \\
-1 & \ldots & \ldots & -1 & 0
\end{array}\right) \in \mathcal{M}_{m}(\mathbb{C})
$$

and

$$
B:=\left(\begin{array}{ccccc}
A & I_{m} & I_{m} & \ldots & I_{m} \\
-I_{m} & A & I_{m} & \ldots & I_{m} \\
\vdots & \ddots & \ddots & \ddots & \vdots \\
-I_{m} & \ldots & -I_{m} & A & I_{m} \\
-I_{m} & \ldots & \ldots & -I_{m} & A
\end{array}\right) \in \mathcal{M}_{m n}(\mathbb{C})
$$

where $I_{m}$ denotes the identity matrix of $\mathcal{M}_{m}$. Then $\Lambda$ is the $m n \times m n$ matrix whose entries are defined by $\Lambda_{k, l}=q^{b_{k, l}}$ for all $k, l \in \llbracket 1, m n \rrbracket$.

It follows from [4, Théorèmes 5.1.1, 5.5.1, 5.5.2] that $\operatorname{Spec}_{\langle 0\rangle}(R)$ is Zariski-homeomorphic to the prime spectrum of the quantum torus $P(\Lambda):=\bar{R} \Sigma^{-1}$, where $\Sigma$ denotes the multiplicative system of $\bar{R}$ generated by the normal elements $T_{i, \alpha}$ with $(i, \alpha) \in \llbracket 1, m \rrbracket \times \llbracket 1, n \rrbracket$. Next, $\operatorname{Spec}(P(\Lambda))$ is Zariski-homeomorphic via extension and contraction to the prime spectrum of the centre $Z(P(\Lambda))$ of $P(\Lambda)$, by [8, Corollary 1.5]. Further, $Z(P(\Lambda))$ turns out to be a Laurent polynomial ring. To make this result precise, we need to introduce the following notation.

If $\underline{s}=\left(s_{1,1}, s_{1,2}, \ldots, s_{m, n}\right) \in \mathbb{Z}^{m n}$, then we set $T^{\underline{s}}:=T_{1,1}^{s_{1,1}} T_{1,2}^{s_{1,2}} \ldots T_{m, n}^{s_{m, n}} \in P(\Lambda)$.

As in [8], we denote by $\sigma: \mathbb{Z}^{m n} \times \mathbb{Z}^{m n} \rightarrow \mathbb{C}^{*}$ the antisymmetric bicharacter defined by

$$
\sigma(\underline{s}, \underline{t}):=\prod_{k, l=1}^{m n} \Lambda_{k, l}^{s_{k} t_{l}} \quad \text { for all } \quad \underline{s}, \underline{t} \in \mathbb{Z}^{m n} .
$$

Then it follows from [8, 1.3] that the centre $Z(P(\Lambda))$ of $P(\Lambda)$ is a Laurent polynomial ring in the variables $\left(T^{\underline{b_{1}}}\right)^{ \pm 1}, \ldots,\left(T_{\underline{b_{r}}}\right)^{ \pm 1}$, where $\left(\underline{b_{1}}, \ldots, \underline{b_{r}}\right)$ is any basis of $S:=\left\{\underline{s} \in \mathbb{Z}^{m n} \mid\right.$ $\sigma(\underline{s},-) \equiv 1\}$. Since $q$ is not a root of unity, easy computations show that $\underline{s} \in S$ if and only if $B^{t} \underline{s}^{t}=0$. Hence the centre $Z(P(\Lambda))$ of $P(\Lambda)$ is a Laurent polynomial ring in $\operatorname{ker}\left(B^{t}\right)$ indeterminates; so we have just proved the following statement.

Proposition 1.2 $\operatorname{Spec}_{\langle 0\rangle}\left(\mathcal{O}_{q}\left(M_{m, n}\right)\right)$ is Zariski-homeomorphic to the prime spectrum of a commutative Laurent polynomial ring in $\operatorname{dim}\left(\operatorname{ker}\left(B^{t}\right)\right)=\operatorname{dim}(\operatorname{ker}(B))$ indeterminates. 
We deduce from Propositions 1.1 and 1.2 the following criterion for $R$ to be a primitive ring.

Corollary $1.3 R$ is a primitive ring if and only if $B$ is invertible.

We now compute the dimension of the kernel of $B$. First, straightforward computations show that

$$
\operatorname{dim}(\operatorname{ker}(B))=m-\operatorname{rk}\left[(A+I)^{n}+(A-I)^{n}\right]
$$

If $i$ is a positive integer greater than or equal to 2 , we denote by $v_{2}(i)$ the 2 -adic valuation of $i$; that is, $m$ is the largest integer such that $2^{m} \mid i$.

One can easily check the following result.

\section{Proposition 1.4}

$$
\operatorname{dim}(\operatorname{ker}(B))=\left\{\begin{array}{cc}
0 & \text { if } v_{2}(m) \neq v_{2}(n) \\
m \wedge n & \text { otherwise }
\end{array}\right.
$$

where $m \wedge n$ denotes the greatest common divisor of $m$ and $n$.

Proof. Set $e_{k}:=\exp \left(i\left(\frac{(2 k+1) \pi}{m}\right)\right)$ for all $k \in \llbracket 0, m-1 \rrbracket$; that is, the $e_{k}$ are the $m$ th root of -1 . It is easy to show that there exists $U \in \mathrm{GL}_{m}(\mathbb{C})$ such that $U^{-1} A U=$ $\operatorname{diag}\left(\frac{e_{0}+1}{e_{0}-1}, \ldots, \frac{e_{m-1}+1}{e_{m-1}-1}\right)$. Hence we have

$$
\begin{aligned}
& U^{-1}\left[(A+I)^{n}+(A-I)^{n}\right] U= \\
& \quad \operatorname{diag}\left(\left(\frac{2 e_{0}}{e_{0}-1}\right)^{n}+\left(\frac{2}{e_{0}-1}\right)^{n}, \ldots,\left(\frac{2 e_{m-1}}{e_{m-1}-1}\right)^{n}+\left(\frac{2}{e_{m-1}-1}\right)^{n}\right) .
\end{aligned}
$$

Since $U \in \mathrm{GL}_{m}(\mathbb{C})$,

$$
\begin{aligned}
\operatorname{rk}\left((A+I)^{n}+(A-I)^{n}\right) & =\operatorname{rk}\left(U^{-1}\left[(A+I)^{n}+(A-I)^{n}\right] U\right) \\
& =\left|\left\{k \in \llbracket 0, m-1 \rrbracket \mid\left(\frac{2 e_{k}}{e_{k}-1}\right)^{n}+\left(\frac{2}{e_{k}-1}\right)^{n} \neq 0\right\}\right|
\end{aligned}
$$

so that

$$
\operatorname{dim}(\operatorname{ker}(B))=\left|\left\{k \in \llbracket 0, m-1 \rrbracket \mid\left(\frac{2 e_{k}}{e_{k}-1}\right)^{n}+\left(\frac{2}{e_{k}-1}\right)^{n}=0\right\}\right|
$$

Now, easy calculations show that $\left(\frac{2 e_{k}}{e_{k}-1}\right)^{n}+\left(\frac{2}{e_{k}-1}\right)^{n}=0$ if and only if $e_{k}^{n}=-1$; that is, if and only if $\exp \left(i\left(\frac{(2 k+1) n \pi}{m}\right)\right)=-1$, that is, if and only if $m \mid(2 k+1) n$ and $\frac{(2 k+1) n}{m}$ is an odd integer. Hence,

$$
\operatorname{dim}(\operatorname{ker}(B))=\mid\left\{k \in \llbracket 0, m-1 \rrbracket|m|(2 k+1) n \text { and } \frac{(2 k+1) n}{m} \text { is odd }\right\} \mid
$$


We now distinguish between two cases.

- First, assume that $v_{2}(m) \neq v_{2}(n)$. Suppose that there exists $k \in \llbracket 0, m-1 \rrbracket$ such that $m \mid(2 k+1) n$. Then $v_{2}(m) \leq v_{2}(n)$. This implies that $v_{2}(m)<v_{2}(n)$, since $v_{2}(m) \neq v_{2}(n)$; and so $\frac{(2 k+1) n}{m}$ is even. Hence there does not exist $k \in \llbracket 0, m-1 \rrbracket$ such that $m \mid(2 k+1) n$ and $\frac{(2 k+1) n}{m}$ is odd. This proves that, in this case,

$$
\operatorname{dim}(\operatorname{ker}(B))=\mid\left\{k \in \llbracket 0, m-1 \rrbracket|m|(2 k+1) n \text { and } \frac{(2 k+1) n}{m} \text { is odd }\right\} \mid=0,
$$

as desired.

- Next, assume that $v_{2}(m)=v_{2}(n)=\alpha$. Write $n=2^{\alpha} v$ and $m=2^{\alpha} u$ with $u$, $v$ odd. It follows that

$$
\begin{aligned}
\operatorname{dim}(\operatorname{ker}(B)) & =\mid\left\{k \in \llbracket 0, m-1 \rrbracket|u|(2 k+1) v \text { and } \frac{(2 k+1) v}{u} \text { is odd }\right\} \mid \\
& =|\{k \in \llbracket 0, m-1 \rrbracket|u|(2 k+1) v\}|
\end{aligned}
$$

Set $d^{\prime}=u \wedge v$ and $u=d^{\prime} u^{\prime}$. Note that $d^{\prime}$ and $u^{\prime}$ are odd, since $u$ and $v$ are odd. This implies that $\operatorname{dim}(\operatorname{ker}(B))=\left|\left\{k \in \llbracket 0, m-1 \rrbracket\left|u^{\prime}\right|(2 k+1)\right\}\right|$, the number of odd multiples of $u^{\prime}$ less that $2 m-1=2^{\alpha+1} d^{\prime} u^{\prime}-1$. Hence $\operatorname{dim}(\operatorname{ker}(B))=2^{\alpha} d^{\prime}=m \wedge n$, as required.

Proposition 1.4 together with Proposition 1.2 and Corollary 1.3 yields the following results.

Theorem 1.5 $\operatorname{Spec}_{\langle 0\rangle}\left(\mathcal{O}_{q}\left(M_{m, n}\right)\right)$ is Zariski-homeomorphic to the prime spectrum of a commutative Laurent polynomial ring in $\alpha_{m, n}$ indeterminates, where

$$
\alpha_{m, n}=\left\{\begin{array}{cc}
0 & \text { if } v_{2}(m) \neq v_{2}(n) \\
m \wedge n & \text { otherwise. }
\end{array}\right.
$$

Theorem $1.6 \mathcal{O}_{q}\left(M_{m, n}\right)$ is a primitive ring if and only if $v_{2}(m) \neq v_{2}(n)$.

For example, $\mathcal{O}_{q}\left(M_{2}\right)$ is not primitive, while $\mathcal{O}_{q}\left(M_{2,3}\right)$ is primitive.

\section{Height one primes in quantum matrices.}

In this section, we investigate height one primes of $R=\mathcal{O}_{q}\left(M_{m, n}\right)$. Every height one prime ideal of $R$ is generated by a normal element, since $R$ is a Noetherian UFD, 14. In this section, we describe explicitly the normal elements that generate the height one prime ideals. Some of them are already known. Indeed, the height one primes that are $\mathcal{H}$-invariant have been described in [14]. Hence, we mainly focus our attention on the other height one primes: these belong to the $\langle 0\rangle$-stratum of $\operatorname{Spec}\left(\mathcal{O}_{q}\left(M_{m, n}\right)\right)$. 


\subsection{Height one primes of $\mathcal{O}_{q}\left(M_{m, n}\right)$ that are $\mathcal{H}$-invariant.}

The algebra $\mathcal{O}_{q}\left(M_{n}\right)$ has a special element, $\operatorname{det}_{q}$, the quantum determinant, defined by

$$
\operatorname{det}_{q}:=\sum_{\sigma}(-q)^{l(\sigma)} Y_{1, \sigma(1)} \cdots Y_{n, \sigma(n)}
$$

where the sum is taken over the permutations of $\{1, \ldots, n\}$ and $l(\sigma)$ is the usual length function on such permutations. The quantum determinant is a central element of $\mathcal{O}_{q}\left(M_{n}\right)$, see, for example, [17, Theorem 4.6.1]. If $I$ is a $t$-element subset of $\{1, \ldots, m\}$ and $\Gamma$ is a $t$ element subset of $\{1, \ldots, n\}$, then the quantum determinant of the subalgebra of $\mathcal{O}_{q}\left(M_{m, n}\right)$ generated by $Y_{i, \alpha}$, with $i \in I$ and $\alpha \in \Gamma$, is denoted by $[I \mid \Gamma]$. The elements $[I \mid \Gamma]$ are the quantum minors of $\mathcal{O}_{q}\left(M_{m, n}\right)$. Note that the quantum minors are $\mathcal{H}$-eigenvectors; and so every ideal generated by a family of quantum minors is $\mathcal{H}$-invariant.

It follows from [14, Proposition 4.2] that there are exactly $m+n-1 \mathcal{H}$-invariant prime ideals in $R$ that have height one. To make this result precise, let us introduce some notation.

For $1 \leq i \leq n+m-1$, let $b_{i}$ be the quantum minor defined as follows.

$$
b_{i}:= \begin{cases}{[1, \ldots, i \mid n-i+1, \ldots, n]} & \text { if } 1 \leq i \leq m \\ {[1, \ldots, m \mid n-i+1, \ldots, n+m-i]} & \text { if } m<i \leq n \\ {[i-n+1, \ldots, m \mid 1, \ldots, m+n-i]} & \text { if } n<i \leq m+n-1\end{cases}
$$

Note that the $b_{i}$ with $m \leq i \leq n$ are precisely the $m \times m$ minors of $\mathcal{O}_{q}\left(M_{n}\right)$ that have consecutive column indices. They are homogeneous of degree $m$. This fact will be used several times later.

Proposition 2.1 ([14], Proposition 4.2) There are precisely $m+n-1$ height one prime ideals that are $\mathcal{H}$-invariant in $\mathcal{O}_{q}\left(M_{m, n}\right)$. They are the ideals generated by $b_{1}, \ldots, b_{m+n-1}$.

The quantum minors $b_{i}$ are normal elements of $R$. Moreover they belong to the algebra $\bar{R}$ obtained from $R$ by the standard deleting derivations algorithm, see Section 1.2. Indeed, every quantum minor $b_{i}$ can be expressed as a product of the canonical generators $T_{i, \alpha}$ of $\bar{R}$ as follows.

Lemma 2.2 For $1 \leq i \leq m+n-1$, we have

$$
b_{i}= \begin{cases}T_{1, n-i+1} T_{2, n-i+2} \ldots T_{i, n} & \text { if } 1 \leq i \leq m \\ T_{1, n-i+1} T_{2, n-i+2} \ldots T_{m, n+m-i} & \text { if } m<i \leq n \\ T_{i-n+1,1} T_{i-n+2,2} \ldots T_{m, m+n-i} & \text { if } n<i \leq n+m-1\end{cases}
$$


Proof. This lemma is a consequence of [ 5 , Proposition 5.2.2].

Recall that two elements $a, b$ of $\operatorname{Frac}(R)=\operatorname{Frac}(\bar{R})$ are said to $q$-commute if there exists an integer $\bullet$ such that $a b=q^{\bullet} b a$. Since the $T_{i, \alpha} q$-commute pairwise, it follows from the previous Lemma 2.2 that the $b_{j} q$-commute with the $T_{i, \alpha}$ and that the $b_{j}$ also $q$-commute pairwise. Sometimes, it will not be necessary to know exactly the integers that appear in the power of $q$. However, at some points, we will need the following commutation relations that can be easily deduced from Lemma 2.2 and from the commutation relations between the $T_{i, \alpha}$.

Corollary 2.3 Assume that $m<n$. Then,

1. for all $m \leq i<j \leq n$,

$$
b_{i} b_{j}=q^{\alpha_{i j}} b_{j} b_{i}
$$

where $\alpha_{i j}:=|\{n-i+1, \ldots, m+n-i\} \cap\{n-j+1, \ldots, m+n-j\}|-m$,

2. for all $i \in\{1, \ldots, m-1\}$,

$$
b_{n} b_{i}=q^{\alpha_{i}} b_{i} b_{n} \quad \text { and } \quad b_{n} b_{m+n-i}=b_{m+n-i} b_{n},
$$

where $\alpha_{i}:=|\{n-i+1, \ldots, n\} \cap\{m+1, \ldots, n\}|$.

\subsection{The $\langle 0\rangle$-stratum of $\operatorname{Spec}\left(\mathcal{O}_{q}\left(M_{m, n}\right)\right)$.}

First, it follows from Theorem 1.5 that the $\langle 0\rangle$-stratum of $\operatorname{Spec}\left(\mathcal{O}_{q}\left(M_{m, n}\right)\right)$ is reduced to $\langle 0\rangle$ when $v_{2}(m) \neq v_{2}(n)$. Thus, throughout this section, we assume that $v_{2}(m)=v_{2}(n)$; so that $\operatorname{Spec}_{\langle 0\rangle}\left(\mathcal{O}_{q}\left(M_{m, n}\right)\right)$ is Zariski-homeomorphic to the prime spectrum of a commutative Laurent polynomial ring in $m \wedge n$ indeterminates. We set $d:=m \wedge n$ and we denote by $m^{\prime}$ and $n^{\prime}$ the positive integers such that $m=d m^{\prime}$ and $n=d n^{\prime}$. Observe that $m^{\prime}$ and $n^{\prime}$ are odd, since $v_{2}(m)=v_{2}(n)$. This observation will be crucial in what follows.

In Section 1.2, we have shown that $\operatorname{Spec}_{\langle 0\rangle}(R)$ is Zariski-homeomorphic to the prime spectrum of the quantum torus $P(\Lambda)=\bar{R} \Sigma^{-1}$, where $\Sigma$ denotes the multiplicative system of $\bar{R}$ generated by the normal elements $T_{i, \alpha}$ with $(i, \alpha) \in \llbracket 1, m \rrbracket \times \llbracket 1, n \rrbracket$. Moreover, $\operatorname{Spec}(P(\Lambda))$ is Zariski-homeomorphic via extension and contraction to the prime spectrum of the centre $Z(P(\Lambda))$ of $P(\Lambda)$, by [8, Corollary 1.5]. Before describing the $\langle 0\rangle$-stratum of $R$, we calculate the centre $Z(P(\Lambda))$ of the quantum torus $P(\Lambda)$ 


\subsubsection{The centre of the quantum torus $P(\Lambda)=\bar{R} \Sigma^{-1}$}

Recall that the quantum minors $b_{i}$ belong to $\bar{R}$. Moreover, the $b_{i}$ are invertible in the quantum torus $P(\Lambda)=\bar{R} \Sigma^{-1}$,

For $j \in\{1, \ldots, d\}$, set

$$
\Delta_{j}:=\prod_{i=0}^{m^{\prime}+n^{\prime}-1} b_{i d+j}^{(-1)^{i}}
$$

(Here we set $b_{m+n}:=1$.)

Theorem 2.4 $Z(P(\Lambda))=\mathbb{C}\left[\Delta_{1}^{ \pm 1}, \ldots, \Delta_{d}^{ \pm 1}\right]$

Proof. First, straightforward computations (see also [16, Theorem 2.13]) show that $\Delta_{1}, \ldots, \Delta_{d}$ are central in $P(\Lambda)$, so that $Z(P(\Lambda)) \supseteq \mathbb{C}\left[\Delta_{1}^{ \pm 1}, \ldots, \Delta_{d}^{ \pm 1}\right]$.

If $\underline{s}=\left(s_{1,1}, s_{1,2}, \ldots, s_{m, n}\right) \in \mathbb{Z}^{m n}$, we set $T^{\underline{s}}:=T_{1,1}^{s_{1,1}} T_{1,2}^{s_{1,2}} \ldots T_{m, n}^{s_{m, n}} \in P(\Lambda)$. Since the $T_{i, \alpha} q$-commute, we deduce from Lemma 2.2 that the central elements $\Delta_{i}$ can be expressed as follows:

$$
\Delta_{i}=q^{\gamma_{i}} T \stackrel{u^{(i)}}{ }
$$

where $\gamma_{i} \in \mathbb{Z}$ and $\underline{u^{(i)}}=\left(u_{1,1}^{(i)}, u_{1,2}^{(i)}, \ldots, u_{m, n}^{(i)}\right) \in \mathbb{Z}^{m n}$ with $\left(u_{1, m-d+1}^{(i)}, u_{1, m-d+2}^{(i)}, \ldots, u_{1, m}^{(i)}\right)=$ $(0, \ldots, 0,1,0, \ldots, 0)$ (the one being in the $i$-th position).

As in [8], we denote by $\sigma: \mathbb{Z}^{m n} \times \mathbb{Z}^{m n} \rightarrow \mathbb{C}^{*}$ the antisymmetric bicharacter defined by

$$
\sigma(\underline{s}, \underline{t})=\prod_{k, l=1}^{m n} \Lambda_{k, l}^{s_{k} t_{l}}
$$

for all $\underline{s}, \underline{t} \in \mathbb{Z}^{m n}$.

Then it follows from [8, 1.3] that the centre $Z(P(\Lambda))$ of $P(\Lambda)$ is a Laurent polynomial ring in the variables $\left(T^{b_{1}}\right)^{ \pm 1}, \ldots,\left(T^{\underline{b_{r}}}\right)^{ \pm 1}$, where $\left(\underline{b_{1}}, \ldots, \underline{b_{r}}\right)$ is any basis of $S:=\left\{\underline{s} \in \mathbb{Z}^{m n} \mid\right.$ $\sigma(\underline{s},-) \equiv 1\}$.

Now, because of Theorem 1.5, we know $r=\operatorname{rk}(S)=d$. Moreover, the $\Delta_{i}$ are central, so that the $\underline{u^{(i)}}$ belong to $S$. To conclude, observe that, since we have

$$
\left(u_{1, m-d+1}^{(i)}, u_{1, m-d+2}^{(i)}, \ldots, u_{1, m}^{(i)}\right)=(0, \ldots, 0,1,0, \ldots, 0),
$$

the group $\mathbb{Z}^{m n} / \sum_{i=1}^{d} \mathbb{Z} \underline{u^{(i)}}$ is torsionfree. Hence, the $\underline{u^{(i)}}$ form a basis of $S$, and so the centre $Z(P(\Lambda))$ of $P(\Lambda)$ is a Laurent polynomial ring in the variables $\Delta_{1}^{ \pm 1}, \ldots, \Delta_{d}^{ \pm 1}$, as desired. 


\subsubsection{Height one primes in $\mathcal{O}_{q}\left(M_{m, n}\right)$.}

Let $P$ a height one prime of $R=\mathcal{O}_{q}\left(M_{m, n}\right)$. Because of the $\mathcal{H}$-stratification, see (II) in Section 1.1, there exists an $\mathcal{H}$-invariant prime ideal $J$ of $R$ such that $P$ belongs to the $\mathcal{H}$-stratum associated to $J$. In particular, we have $J \subseteq P$. Since $P$ has height one, this implies that the height of $J$ is at most 1 . Thus, two cases arise.

1. First, suppose that $J$ has height one. In this case, $P=J$, since $P$ has height one; so that $P$ is an $\mathcal{H}$-invariant height one prime ideal of $R$. Hence it follows from Proposition 2.1] that there exists $i \in\{1, \ldots, m+n-1\}$ such that $P=\left\langle b_{i}\right\rangle$.

2. Next, suppose that $J=\langle 0\rangle$. In this case, $P$ is a height one prime ideal that belongs to the $\langle 0\rangle$-stratum of $\operatorname{Spec}(R)$. Note that this case can only arise when $v_{2}(m)=v_{2}(n)$, since it follows from Theorem 1.5 that the $\langle 0\rangle$-stratum of $\operatorname{Spec}\left(\mathcal{O}_{q}\left(M_{m, n}\right)\right)$ is reduced to $\langle 0\rangle$ when $v_{2}(m) \neq v_{2}(n)$.

Let $\operatorname{Spec}^{1}(R)$ denote the set of all height one primes of $R$. The previous discussion proves the following statement.

\section{Proposition 2.5}

1. If $v_{2}(m) \neq v_{2}(n)$, then

$$
\operatorname{Spec}^{1}(R)=\left\{\left\langle b_{i}\right\rangle \mid i \in\{1, \ldots, m+n-1\}\right\} .
$$

2. If $v_{2}(m)=v_{2}(n)$, then

$$
\operatorname{Spec}^{1}(R)=\left\{\left\langle b_{i}\right\rangle \mid i \in\{1, \ldots, m+n-1\}\right\} \cup \operatorname{Spec}_{\langle 0\rangle}^{1}(R),
$$

where $\operatorname{Spec}_{\langle 0\rangle}^{1}(R)$ denotes the set of all height one primes of $R$ that belong to the $\langle 0\rangle$-stratum of $\operatorname{Spec}(R)$.

In order to complete the previous result, we now describe, in the case where $v_{2}(m)=$ $v_{2}(n)$, the height one primes of $\mathcal{O}_{q}\left(M_{m, n}\right)$ that belongs to $\operatorname{Spec}_{\langle 0\rangle}\left(\mathcal{O}_{q}\left(M_{m, n}\right)\right)$.

Proposition 2.6 Assume that $v_{2}(m)=v_{2}(n)$. Then, for any height one prime ideal $P$ of $\mathcal{O}_{q}\left(M_{m, n}\right)$ that belongs to $\operatorname{Spec}_{\langle 0\rangle}\left(\mathcal{O}_{q}\left(M_{m, n}\right)\right)$, there exists a unique (up to scalar) irreducible 
polynomial $V=\sum_{i_{1}=0}^{r_{1}} \cdots \sum_{i_{d}=0}^{r_{d}} a_{i_{1}, \ldots, i_{d}} X_{1}^{i_{1}} \ldots X_{d}^{i_{d}} \in \mathbb{C}\left[X_{1}, \ldots, X_{d}\right]$ (where $r_{i}=\operatorname{deg}_{X_{i}} V$ ) with $V \neq X_{i}$ for all $i \in\{1, \ldots, d\}$ such that $P=\langle u\rangle$ where

$$
u:=\sum_{i_{1}=0}^{r_{1}} \cdots \sum_{i_{d}=0}^{r_{d}} a_{i_{1}, \ldots, i_{d}} \prod_{j=1}^{d}\left[\prod_{\substack{i=0 \\ i \text { even }}}^{m^{\prime}+n^{\prime}-1} b_{i d+j}\right]^{i_{j}}\left[\prod_{\substack{i=0 \\ i \text { odd }}}^{m^{\prime}+n^{\prime}-1} b_{i d+j}\right]^{r_{j}-i_{j}} .
$$

Moreover, $u$ is normal in $R$.

Proof. We proceed in three steps.

- Step 1. A generator for the extension of $P$ in a localisation of $R$.

First, observe that the prime ideals in $\operatorname{Spec}_{\langle 0\rangle}(R)$ do not contain any $b_{i}$. Indeed, assume that this is not the case; that is, assume that there exists $P \in \operatorname{Spec}_{\langle 0\rangle}(R)$ with $b_{i} \in P$ for a certain $i$. Then, since $b_{i}$ is an $\mathcal{H}$-eigenvector, we have $b_{i} \in \bigcap_{h \in \mathcal{H}} h . P=\langle 0\rangle$. This is a contradiction; and so $\operatorname{Spec}_{\langle 0\rangle}(R) \subseteq\left\{P \in \operatorname{Spec}(R) \mid b_{i} \notin P\right.$ for all $\left.i\right\}$. On the other hand, if $P$ is a prime ideal of $R$ such that $b_{i} \notin P$ for all $i$, then $\bigcap_{h \in \mathcal{H}} h . P$ is an $\mathcal{H}$-invariant prime ideal of $R$ that does not contain any $b_{i}$. However, because of [14, Proposition 2.9], every nonzero $\mathcal{H}$-invariant prime ideal of $R$ contains a height one prime that is $\mathcal{H}$ invariant. In other words, every nonzero $\mathcal{H}$-invariant prime ideal of $R$ contains a $b_{i}$. Thus $\bigcap_{h \in \mathcal{H}} h . P=\langle 0\rangle$ and so $P \in \operatorname{Spec}_{\langle 0\rangle}(R)$. To sum up, we have shown that $\operatorname{Spec}_{\langle 0\rangle}(R)=$ $\left\{P \in \operatorname{Spec}(R) \mid b_{i} \notin P\right.$ for all $\left.i\right\}$.

Denote by $T$ the localisation of $R$ with respect of the multiplicatively closed set $\mathcal{B}$ generated by the normal elements $b_{i}$. Note that the torus $\mathcal{H}$ still acts rationally by automorphisms on $T$, since the $b_{i}$ are $\mathcal{H}$-eigenvectors, see [3, Exercise II.3.A]. Moreover, it follows from the previous study, and from classical results of non-commutative localisation theory, that the map $\varphi: P \rightarrow P \mathcal{B}^{-1}$ is an increasing bijection from $\operatorname{Spec}_{\langle 0\rangle}(R)$ onto $\operatorname{Spec}(T)$.

Next, $T$ is $\mathcal{H}$-simple; that is, the only $\mathcal{H}$-invariant ideals in $T$ are $\langle 0\rangle$ and $T$, by [14, Proposition 3.5]. The action of $\mathcal{H}$ on $T$ is rational, see [3, Exercise II.3.A], and $T$ is $\mathcal{H}$-simple; so [3, Corollary II.3.9] applies to $T$. Thus extension and contraction provide mutually inverse bijections between $\operatorname{Spec}(T)$ and $\operatorname{Spec}(Z(T))$.

Now, recall that the $b_{i}$ can be expressed as products of $T_{i, \alpha}$, see Lemma 2.2. so the $b_{i}$ belong to the quantum torus $P(\Lambda)=\bar{R} \Sigma^{-1}$. Moreover, it follows from [4, Théorème 3.3.1] that there exists a multiplicative system $S$ of $R$ such that $R \subseteq R S^{-1}=P(\Lambda)=\bar{R} \Sigma^{-1}$. Hence $T$ is a subalgebra of the quantum torus $P(\Lambda)=R S^{-1}$, and $R \subseteq T \subseteq P(\Lambda)=$ 
$\bar{R} \Sigma^{-1}=R S^{-1}$. Thus $Z(T) \subseteq Z(P(\Lambda))=\mathbb{C}\left[\Delta_{1}^{ \pm 1}, \ldots, \Delta_{d}^{ \pm 1}\right]$. But the $\Delta_{i}$ are just products of $b_{i}^{ \pm 1}$, so that they belong to $T$. Hence $Z(T)=\mathbb{C}\left[\Delta_{1}^{ \pm 1}, \ldots, \Delta_{d}^{ \pm 1}\right]$.

Observe that $\Delta_{j}$ can be written as follows.

$$
\Delta_{j}=q^{\bullet} \prod_{\substack{i=0 \\ i \text { even }}}^{m^{\prime}+n^{\prime}-1} b_{i d+j} \prod_{\substack{i=0 \\ i \text { odd }}}^{m^{\prime}+n^{\prime}-1} b_{i d+j}^{-1}
$$

where as usual $\bullet$ denotes an integer, since the $b_{i} q$-commute. For $j \in\{1, \ldots, d\}$, we set

$$
\Delta_{j}^{\prime}=\left(\prod_{\substack{i=0 \\ i \text { even }}}^{m^{\prime}+n^{\prime}-1} b_{i d+j}\right)\left(\prod_{\substack{i=0 \\ i \text { odd }}}^{m^{\prime}+n^{\prime}-1} b_{i d+j}\right)^{-1}
$$

Since $Z(T)=\mathbb{C}\left[\Delta_{1}^{ \pm 1}, \ldots, \Delta_{d}^{ \pm 1}\right]$, the centre of $T$ is also the (commutative) Laurent polynomial ring in the indeterminates $\Delta_{1}^{\prime}, \ldots, \Delta_{d}^{\prime}$, that is,

$$
Z(T)=\mathbb{C}\left[\Delta_{1}^{\prime \pm 1}, \ldots, \Delta_{d}^{\prime \pm 1}\right]
$$

Now, let $P$ be a height one prime of $R$ that belongs to $\operatorname{Spec}_{\langle 0\rangle}(R)$. It follows from the previous study that $\varphi(P) \cap Z(T)$ is a height one prime of $Z(T)=\mathbb{C}\left[\Delta_{1}^{\prime \pm 1}, \ldots, \Delta_{d}^{\prime \pm 1}\right]$. Hence there exists an irreducible polynomial $V=\sum_{i_{1}=0}^{r_{1}} \cdots \sum_{i_{d}=0}^{r_{d}} a_{i_{1}, \ldots, i_{d}} X_{1}^{i_{1}} \ldots X_{d}^{i_{d}} \in$ $\mathbb{C}\left[X_{1}, \ldots, X_{d}\right]$, with $r_{i}=\operatorname{deg}_{X_{i}} V$, and with $V \neq X_{i}$ for all $i \in\{1, \ldots, d\}$, such that

$$
\varphi(P) \cap Z(T)=\left\langle\sum_{i_{1}=0}^{r_{1}} \cdots \sum_{i_{d}=0}^{r_{d}} a_{i_{1}, \ldots, i_{d}} \Delta_{1}^{\prime i_{1}} \ldots \Delta_{d}^{\prime i_{d}}\right\rangle_{Z(T)} .
$$

Thus,

$$
\varphi(P)=\left\langle\sum_{i_{1}=0}^{r_{1}} \cdots \sum_{i_{d}=0}^{r_{d}} a_{i_{1}, \ldots, i_{d}} \Delta_{1}^{\prime i_{1}} \ldots \Delta_{d}^{\prime i_{d}}\right\rangle_{T}
$$

since extension and contraction provide mutually inverse bijections between $\operatorname{Spec}(T)$ and $\operatorname{Spec}(Z(T))$. Since the $b_{i}$ are invertible in $T$, (2) leads to $\varphi(P)=\langle u\rangle$, where

$$
u:=\sum_{i_{1}=0}^{r_{1}} \cdots \sum_{i_{d}=0}^{r_{d}} a_{i_{1}, \ldots, i_{d}} \prod_{j=1}^{d}\left[\prod_{\substack{i=0 \\ i \text { even }}}^{m^{\prime}+n^{\prime}-1} b_{i d+j}\right]^{i_{j}}\left[\prod_{\substack{i=0 \\ i \text { odd }}}^{m^{\prime}+n^{\prime}-1} b_{i d+j}\right]^{r_{j}-i_{j}} .
$$

Note that $u$ is a normal element in both $R$ and $T$. 
- Step 2. We prove that u is not contained in any $\left\langle b_{k}\right\rangle$.

Set

$$
u_{i_{1}, \ldots, i_{d}}:=\prod_{j=1}^{d}\left[\prod_{\substack{i=0 \\ i \text { even }}}^{m^{\prime}+n^{\prime}-1} b_{i d+j}\right]^{i_{j}}\left[\prod_{\substack{i=0 \\ i \text { odd }}}^{m^{\prime}+n^{\prime}-1} b_{i d+j}\right]^{r_{j}-i_{j}}
$$

for all $\left(i_{1}, \ldots, i_{d}\right) \in \llbracket 0, r_{1} \rrbracket \times \cdots \times \llbracket 0, r_{d} \rrbracket$; so that

$$
u=\sum_{i_{1}=0}^{r_{1}} \cdots \sum_{i_{d}=0}^{r_{d}} a_{i_{1}, \ldots, i_{d}} u_{i_{1}, \ldots, i_{d}} .
$$

Since the $b_{k}$ are $\mathcal{H}$-eigenvectors, it is clear that the $u_{i_{1}, \ldots, i_{d}}$ are also $\mathcal{H}$-eigenvectors. Hence, for all $\left(i_{1}, \ldots, i_{d}\right) \in \llbracket 0, r_{1} \rrbracket \times \cdots \times \llbracket 0, r_{d} \rrbracket$, there exists a (unique) character $f_{i_{1}, \ldots, i_{d}}: \mathcal{H} \rightarrow \mathbb{C}^{*}$ such that $h . u_{i_{1}, \ldots, i_{d}}=f_{i_{1}, \ldots, i_{d}}(h) u_{i_{1}, \ldots, i_{d}}$ for all $h \in \mathcal{H}$. The character $f_{i_{1}, \ldots, i_{d}}$ is called the $\mathcal{H}$-eigenvalue of the $\mathcal{H}$-eigenvector $u_{i_{1}, \ldots, i_{d}}$.

Claim 2.7 The $u_{i_{1}, \ldots, i_{d}}$ are $\mathcal{H}$-eigenvectors with pairwise distinct $\mathcal{H}$-eigenvalues.

Proof of Claim 2.7. Let $\left(i_{1}, \ldots, i_{d}\right) \in \llbracket 0, r_{1} \rrbracket \times \cdots \times \llbracket 0, r_{d} \rrbracket$ and $h:=\left(g_{1}, \ldots, g_{m} ; h_{1}, \ldots, h_{n}\right) \in$ $\mathcal{H}$. Then,

$$
h . u_{i_{1}, \ldots, i_{d}}=g_{1}^{\bullet} \ldots g_{m}^{\bullet} h_{1}^{\bullet} \ldots h_{n-m}^{\bullet} h_{n-m+1}^{\alpha_{m-1}} \ldots h_{n}^{\alpha_{0}} u_{i_{1}, \ldots, i_{d}},
$$

where $\bullet \in \mathbb{Z}$ and $\alpha_{k}$ is the number of quantum minors involving the $(n-k)$-th column in the product that defines $u_{i_{1}, \ldots, i_{d}}$.

Now one can observe that, for all $k \in\{0, \ldots, d-1\}$, the $(n-k)$-th column appears in $b_{i d+j}$ if and only if either (i) $k+1 \leq j \leq d$ and $0 \leq i \leq m^{\prime}-1$, or (ii) $1 \leq j \leq k$ and $1 \leq i \leq m^{\prime}$.

Hence, since $m^{\prime}$ is odd,

$$
\begin{aligned}
\alpha_{k} & =\sum_{j=1}^{k}\left(\frac{m^{\prime}-1}{2} i_{j}+\frac{m^{\prime}+1}{2}\left(r_{j}-i_{j}\right)\right)+\sum_{j=k+1}^{d}\left(\frac{m^{\prime}+1}{2} i_{j}+\frac{m^{\prime}-1}{2}\left(r_{j}-i_{j}\right)\right) \\
& =\sum_{j=1}^{k}\left(\frac{m^{\prime}+1}{2} r_{j}-i_{j}\right)+\sum_{j=k+1}^{d}\left(\frac{m^{\prime}-1}{2} r_{j}+i_{j}\right)
\end{aligned}
$$

Now, let $\left(i_{1}, \ldots, i_{d}\right),\left(i_{1}^{\prime}, \ldots, i_{d}^{\prime}\right) \in \llbracket 0, r_{1} \rrbracket \times \cdots \times \llbracket 0, r_{d} \rrbracket$, and assume that $u_{i_{1}, \ldots, i_{d}}$ and $u_{i_{1}^{\prime}, \ldots, i_{d}^{\prime}}$ are associated to the same $\mathcal{H}$-eigenvalue. Then, since $\mathbb{C}$ is infinite, it follows from the previous study that,

$$
\sum_{j=1}^{k}\left(\frac{m^{\prime}+1}{2} r_{j}-i_{j}\right)+\sum_{j=k+1}^{d}\left(\frac{m^{\prime}-1}{2} r_{j}+i_{j}\right)=\sum_{j=1}^{k}\left(\frac{m^{\prime}+1}{2} r_{j}-i_{j}^{\prime}\right)+\sum_{j=k+1}^{d}\left(\frac{m^{\prime}-1}{2} r_{j}+i_{j}^{\prime}\right),
$$


for all $k \in\{0, \ldots, d-1\}$. Hence,

$$
\sum_{j=1}^{k}\left(i_{j}^{\prime}-i_{j}\right)+\sum_{j=k+1}^{d}\left(i_{j}-i_{j}^{\prime}\right)=0
$$

for all $k \in\{0, \ldots, d-1\}$. This forces $i_{j}=i_{j}^{\prime}$ for all $j \in\{1, \ldots, d\}$; so the claim is proved.

Next, we prove that $u$ does not belong to any $\left\langle b_{k}\right\rangle$. Indeed, assume that $u \in\left\langle b_{k}\right\rangle$ for a certain $k \in\{1, \ldots, m+n-1\}$. Then, since $\left\langle b_{k}\right\rangle$ is an $\mathcal{H}$-invariant prime ideal of $R$, it follows from the previous claim and from [3, II.2.10] that:

$$
\text { If } a_{i_{1}, \ldots, i_{d}} \neq 0 \text {, then } u_{i_{1}, \ldots, i_{d}}=\prod_{j=1}^{d}\left[\prod_{\substack{i=0 \\ i \text { even }}}^{m^{\prime}+n^{\prime}-1} b_{i d+j}\right]^{i_{j}}\left[\prod_{\substack{i=0 \\ i \text { odd }}}^{m^{\prime}+n^{\prime}-1} b_{i d+j}\right]^{r_{j}-i_{j}} \in\left\langle b_{k}\right\rangle \text {. }
$$

Set $k:=r d+s$ with $r \in\left\{0, \ldots, m^{\prime}+n^{\prime}-1\right\}$ and $s \in\{1, \ldots, d\}$. We distinguish between two cases. First, assume that $r$ is even. Since the $\left\langle b_{i}\right\rangle$ are pairwise distinct height one completely prime ideals of $R$, we deduce from (3) that $a_{i_{1}, \ldots, i_{d}}=0$ if $i_{s}=0$. This implies that $V=\sum_{i_{1}=0}^{r_{1}} \cdots \sum_{i_{d}=0}^{r_{d}} a_{i_{1}, \ldots, i_{d}} X_{1}^{i_{1}} \ldots X_{d}^{i_{d}}=X_{s} V^{\prime}$, with $V^{\prime} \in \mathbb{C}\left[X_{1}, \ldots, X_{d}\right]$. This contradicts the facts that $V$ is irreducible and $V \neq X_{s}$.

Next, assume that $r$ is odd. Since the $\left\langle b_{i}\right\rangle$ are pairwise distinct height one completely prime ideals of $R$, we deduce from (3) that $a_{i_{1}, \ldots, i_{d}}=0$ if $i_{s}=r_{s}$. Now this contradicts $\operatorname{deg}_{X_{s}} V=r_{s}$.

To sum up: $u$ does not belong to any $\left\langle b_{k}\right\rangle$.

- Step 3. We prove that $P$ is generated by $u$.

Recall from step one that $\varphi(P)$ is generated by $u$. Hence, it is clear that $P=\varphi(P) \cap R \supseteq$ $\langle u\rangle$. Let now $x \in P$. It remains to prove that $x \in\langle u\rangle$. There exists $\left(\alpha_{1}, \ldots, \alpha_{m+n-1}\right) \in$ $\mathbb{N}^{m+n-1}$ such that $x b_{1}^{\alpha_{1}} \ldots b_{m+n-1}^{\alpha_{m+n-1}}=u r$ with $r \in R$. Choose such a $\left(\alpha_{1}, \ldots, \alpha_{m+n-1}\right) \in$ $\mathbb{N}^{m+n-1}$ minimal (for the lexicographic order). If $\left(\alpha_{1}, \ldots, \alpha_{m+n-1}\right) \neq 0$, there exists $k$ such that $\alpha_{k} \neq 0$. Then $u r=x b_{1}^{\alpha_{1}} \ldots b_{m+n-1}^{\alpha_{m+n-1}}$ belongs to the completely prime ideal of $R$ generated by $b_{k}$. Hence, $u \in\left\langle b_{k}\right\rangle$ or $r \in\left\langle b_{k}\right\rangle$. Because of step two, the first possibility can not happen. Hence, $r \in\left\langle b_{k}\right\rangle$. Since $b_{k}$ is normal, we can write $r=r^{\prime} b_{k}$ with $r^{\prime} \in R$. Thus $x b_{1}^{\alpha_{1}} \ldots b_{m+n-1}^{\alpha_{m+n-1}}=u r=u r^{\prime} b_{k}$; and so $x b_{1}^{\alpha_{1}} \ldots b_{k}^{\alpha_{k}-1} \ldots b_{m+n-1}^{\alpha_{m+n-1}}=u r^{\prime}$. This contradicts the minimality of $\left(\alpha_{1}, \ldots, \alpha_{m+n-1}\right)$. Hence $\left(\alpha_{1}, \ldots, \alpha_{m+n-1}\right)=0$; and so $x=u r \in\langle u\rangle$, as desired. 


\section{Automorphisms of quantum matrices}

In this section, we investigate the group of automorphisms of $\mathcal{O}_{q}\left(M_{m, n}\right)$. Using graded arguments together with the results of the previous sections, we show that, in the non-square case, every $\mathcal{H}$-invariant height one prime of $\mathcal{O}_{q}\left(M_{m, n}\right)$, except possibly one, is invariant under every automorphism. Next, by using the preferred basis of $\mathcal{O}_{q}\left(M_{m, n}\right)$ introduced in [7], we show that the group of automorphisms of $\mathcal{O}_{q}\left(M_{m, n}\right)$, with $2 \leq m<n$, is isomorphic to the torus $\left(\mathbb{C}^{*}\right)^{m+n-1}$.

In the sequel, we will use several times the following well-known result concerning normal elements of $R$.

Lemma 3.1 Let $u$ and $v$ two nonzero normal elements of $R$ such that $\langle u\rangle=\langle v\rangle$. Then there exist $\lambda, \mu \in \mathbb{C}^{*}$ such that $u=\lambda v$ and $v=\mu u$.

\section{$3.1 q$-commutation, gradings and automorphisms.}

Let $A=\oplus_{i \in \mathbb{N}} A_{i}$ be a $\mathbb{N}$-graded $\mathbb{C}$-algebra with $A_{0}=\mathbb{C}$. Assume that $A$ is a domain generated as an algebra by $x_{1}, \ldots, x_{n}$, and that $A_{1}=\mathbb{C} x_{1} \oplus \cdots \oplus \mathbb{C} x_{n}$. We set $A_{\geq d}:=\oplus_{i \geq d} A_{i}$. The following result was inspired by a result in [1].

Proposition 3.2 Assume that, for all $i \in\{1, \ldots, n\}$, there exist $j \neq i$ and $q_{i j} \neq 1$ such that $x_{i} x_{j}=q_{i j} x_{j} x_{i}$. Let $\sigma$ be an automorphism of $A$ and $x$ be a nonzero homogeneous element of degree $d$ of $A$.

Then $\sigma(x)=y_{d}+y_{>d}$, where $y_{d} \in A_{d} \backslash\{0\}$ and $y_{>d} \in A_{>d}$.

Proof. First, observe that it is sufficient to prove that $\sigma\left(A_{d}\right) \subseteq A_{\geq d}$, for every automorphism $\sigma$ of $A$. Indeed, assume that this is the case, and let $x$ be a nonzero homogeneous element of degree $d$ of $A$. Then we can write $\sigma(x)=y_{d}+y_{>d}$, where $y_{d} \in A_{d}$ and $y_{>d} \in A_{>d}$. If $y_{d}=0$, then $\sigma(x)=y_{>d} \in A_{>d}$, and thus $\sigma^{-1}\left(A_{>d}\right)$ is not contained in $A_{>d}$. This is a contradiction.

Hence it just remains to prove that, for every automorphism $\sigma$ of $A$, we have $\sigma\left(A_{d}\right) \subseteq$ $A_{\geq d}$. Naturally, it is sufficient to prove this result when $x=x_{i}$ is one of the canonical generators of $A$. So let $\sigma$ be an automorphism $\sigma$ of $A$ and $i \in\{1, \ldots, n\}$. We can write

$$
\sigma\left(x_{i}\right)=\alpha_{i}+f_{i}
$$

where $\alpha_{i} \in \mathbb{C}$ and $f_{i} \in A_{\geq 1}$. We have to prove that $\alpha_{i}=0$. 
Now, by hypothesis, there exist $j \in\{1, \ldots, n\}$ and $q_{i j} \neq 1$ such that $x_{i} x_{j}=q_{i j} x_{j} x_{i}$. Set

$$
\sigma\left(x_{j}\right)=f_{j}+g_{j},
$$

where $f_{j} \in A_{t}, f_{j} \neq 0$ and $g_{j} \in A_{>t}$. Applying $\sigma$ to the equality $x_{i} x_{j}=q_{i j} x_{j} x_{i}$, and next identifying the homogeneous part of degree $t$ yields $\alpha_{i} f_{j}=q_{i j} f_{j} \alpha_{i}$. Thus, $\alpha_{i} f_{j}=0$, since $q_{i j} \neq 1$. Now, since $f_{j} \neq 0$, this forces $\alpha_{i}=0$, as desired.

Note that the commutativity hypothesis of the Proposition 3.2 is satisfied by the algebra $R=\mathcal{O}_{q}\left(M_{m, n}\right)$, provided that $n \geq 2$. Indeed, the relations that define $R$ are all quadratic, so that $R=\oplus_{i \in \mathbb{N}} R_{i}$ is a $\mathbb{N}$-graded algebra, the canonical generators $Y_{i, \alpha}$ of $R$ having degree one.

Next, for all $(i, \alpha) \in \llbracket 1, m \rrbracket \times \llbracket 1, n \rrbracket$ and $\beta \neq \alpha$, we have $Y_{i, \alpha} Y_{i, \beta}=q^{\bullet} Y_{i, \beta} Y_{i, \alpha}$ with - $= \pm 1$. Thus, the commutativity hypothesis of Proposition 3.2 is satisfied; and so one can state:

Corollary 3.3 Let $\sigma$ be an automorphism of $R=\mathcal{O}_{q}\left(M_{m, n}\right)$ and $x$ an homogeneous element of degree $d$ of $R$.

Then $\sigma(x)=y_{d}+y_{>d}$, where $y_{d} \in R_{d} \backslash\{0\}$ and $y_{>d} \in R_{>d}$.

Note, for later use, that a $t \times t$ quantum minor of $R$ is a homogeneous element of degree $t$ with respect to this grading of $R$. In the sequel, $R$ will always be endowed with this grading.

\subsection{The action of $\operatorname{Aut}\left(\mathcal{O}_{q}\left(M_{m, n}\right)\right)$ on the set of height one primes: the non-square case.}

Throughout this section, we assume that $m<n$. Our aim in this section is to show that every $\mathcal{H}$-invariant height one prime of $\mathcal{O}_{q}\left(M_{m, n}\right)$, except possibly one, is invariant under every automorphism. In order to do this, we will distinguish between two cases.

\subsubsection{The case where $n \neq 3 m$.}

Throughout this section, we assume that $m<n$ and $n \neq 3 m$. In this case, we first show that the set of all $\mathcal{H}$-invariant height one primes of $R$ is invariant under every automorphism of $R$, that is, we have:

Lemma 3.4 Assume that $n \neq 3 m$. Let $\sigma$ be an automorphism of $R$ and $i \in\{1, \ldots, m+$ $n-1\}$. Then there exists $j \in\{1, \ldots, m+n-1\}$ such that $\sigma\left(\left\langle b_{i}\right\rangle\right)=\left\langle b_{j}\right\rangle$. 
Proof. First, assume that $v_{2}(m) \neq v_{2}(n)$. It follows from Proposition 2.5 that $\left\{\left\langle b_{i}\right\rangle \mid i \in\right.$ $\llbracket 1, m+n-1 \rrbracket\}$ is exactly the set of all height one primes of $R$ and so the result is obvious in this case.

Next, assume that $v_{2}(m)=v_{2}(n)$. We use the notation of Section 2.2. In particular, $d$ denotes the greatest common divisor of $m$ and $n$, and we set $m=d m^{\prime}$ and $n=d n^{\prime}$. Observe that $m^{\prime}$ and $n^{\prime}$ are odd. Note further that, since $n \neq 3 m$, we have $\left(m^{\prime}, n^{\prime}\right) \neq(1,3)$. Moreover, since $m<n$, we have $m^{\prime}<n^{\prime}$. Since $m^{\prime}$ and $n^{\prime}$ are both odd, this forces $n^{\prime} \geq 5$.

Since $\left\langle b_{i}\right\rangle$ is a height one prime ideal of $R$, its image under $\sigma$ is also a height one prime ideal of $R$. We distinguish between two cases.

First, if $\left\langle\sigma\left(b_{i}\right)\right\rangle$ is $\mathcal{H}$-invariant, then it follows from Proposition 2.1 that $\left\langle\sigma\left(b_{i}\right)\right\rangle=\left\langle b_{j}\right\rangle$ for some $j$ and so the proof is complete in this case.

Next, assume that $\left\langle\sigma\left(b_{i}\right)\right\rangle$ is not $\mathcal{H}$-invariant. In this case, $\left\langle\sigma\left(b_{i}\right)\right\rangle$ is a height one (completely) prime ideal of $R$ which is not $\mathcal{H}$-invariant. Thus, $\left\langle\sigma\left(b_{i}\right)\right\rangle$ belongs to the $\langle 0\rangle$ stratum of the prime spectrum of $R$, by Proposition 2.5. and so we deduce from Proposition 2.6 that there exist $\left(r_{1}, \ldots, r_{d}\right) \in \mathbb{N}^{d} \backslash\{0\}$ and scalars $a_{i_{1}, \ldots, i_{d}} \in \mathbb{C}$ such that $\left\langle\sigma\left(b_{i}\right)\right\rangle=\langle u\rangle$, where

$$
u:=\sum_{i_{1}=0}^{r_{1}} \cdots \sum_{i_{d}=0}^{r_{d}} a_{i_{1}, \ldots, i_{d}} \prod_{j=1}^{d}\left[\prod_{\substack{i=0 \\ i \text { even }}}^{m^{\prime}+n^{\prime}-1} b_{i d+j}\right]^{i_{j}}\left[\prod_{\substack{i=0 \\ i \text { odd }}}^{m^{\prime}+n^{\prime}-1} b_{i d+j}\right]^{r_{j}-i_{j}} .
$$

It follows from Proposition 2.6 that $u$ is a normal element of $R$. On the other hand, $\sigma\left(b_{i}\right)$ is normal, since $b_{i}$ is normal. Thus, we deduce from Lemma 3.1 that $u \in \mathbb{C}^{*} \sigma\left(b_{i}\right)$.

Now $b_{i}$ is a quantum minor of $R=\mathcal{O}_{q}\left(M_{m, n}\right)$, and so $b_{i}$ is a homogeneous element of degree less than or equal to $m$. So Corollary 3.3 implies that

$$
u \notin R_{>m} .
$$

On the other hand, since $\left(r_{1}, \ldots, r_{d}\right) \neq 0$, there exists $k$ such that $r_{k} \geq 1$. Now, recalling that $m^{\prime}$ and $n^{\prime}$ are odd and that $n^{\prime} \geq 5$, we have:

$\operatorname{deg}\left(\prod_{j=1}^{d}\left[\prod_{\substack{i=0 \\ i \text { even }}}^{m^{\prime}+n^{\prime}-1} b_{i d+j}\right]^{i_{j}}\left[\prod_{\substack{i=0 \\ i \text { odd }}}^{m^{\prime}+n^{\prime}-1} b_{i d+j}\right]^{r_{j}-i_{j}}\right) \geq \begin{cases}\operatorname{deg}\left(b_{k} b_{\left(m^{\prime}+1\right) d+k}\right) & \text { if } i_{k} \geq 1 \\ \operatorname{deg}\left(b_{m^{\prime} d+k} b_{\left(m^{\prime}+n^{\prime}-3\right) d+k}\right) & \text { if } i_{k}=0\end{cases}$

Since $m<n$, we have $m^{\prime}<n^{\prime}$. Further, $m^{\prime}$ and $n^{\prime}$ are odd, and so $m^{\prime}+1<n^{\prime}$. Hence $m=m^{\prime} d \leq m^{\prime} d+k \leq\left(m^{\prime}+1\right) d+k \leq n=n^{\prime} d$. This implies that $b_{\left(m^{\prime}+1\right) d+k}$ and $b_{m^{\prime} d+k}$ 
are $m \times m$ quantum minors. Thus, in both cases, we get

$$
\operatorname{deg}\left(\prod_{j=1}^{d}\left[\prod_{\substack{i=0 \\ i \text { even }}}^{m^{\prime}+n^{\prime}-1} b_{i d+j}\right]^{i_{j}}\left[\prod_{\substack{i=0 \\ i \text { odd }}}^{m^{\prime}+n^{\prime}-1} b_{i d+j}\right]^{r_{j}-i_{j}}\right) \geq m+1 .
$$

Thus $u$ is a linear combination of terms of degree greater than $m$. This implies that $u \in R_{>m}$, contradicting (4); and so the proof is complete.

In fact, more is true: each $\mathcal{H}$-invariant height one prime is left invariant by any automorphism, as the following result shows.

Proposition 3.5 Assume that $n \neq 3 m$ and let $\sigma$ be an automorphism of $R$. Then, for each $i \in\{1, \ldots, m+n-1\}$, there exists $\lambda_{i} \in \mathbb{C}^{*}$ such that $\sigma\left(b_{i}\right)=\lambda_{i} b_{i}$.

Proof. Let $i \in\{1, \ldots, m+n-1\}$. It follows from Lemma 3.4 that there exists $j \in$ $\{1, \ldots, m+n-1\}$ such that $\sigma\left(\left\langle b_{i}\right\rangle\right)=\left(\left\langle b_{j}\right\rangle\right)$. Since $\sigma\left(b_{i}\right)$ and $b_{j}$ are normal, it follows from Lemma 3.1 that there exists $\lambda_{j} \in \mathbb{C}^{*}$ such that $\sigma\left(b_{i}\right)=\lambda_{i} b_{j}$.

Thus, there exist scalars $\lambda_{1}, \ldots, \lambda_{m+n-1} \in \mathbb{C}^{*}$ and a permutation $s \in S_{m+n-1}$ such that $\sigma\left(b_{i}\right)=\lambda_{i} b_{s(i)}$ for all $i \in\{1, \ldots, m+n-1\}$.

We will now prove that $s$ is the identity, that is, $s(i)=i$ for all $i \in\{1, \ldots, m+n-1\}$.

First, let $i \in\{m, \ldots, n\}$, so that $b_{i}$ is a $m \times m$ quantum minor. Then $b_{i} \in R_{m}$ and it follows from Corollary 3.3 that $\sigma\left(b_{i}\right) \in R_{\geq m}$. Hence $b_{s(i)} \in R_{\geq m}$. This implies that $b_{s(i)}$ is also a $m \times m$ quantum minor, so that $s(i) \in\{m, \ldots, n\}$. Thus $s$ induces a permutation of $\{m, \ldots, n\}$.

We now prove with the help of a decreasing induction that $s(i)=i$ for all $i \in\{m, \ldots, n\}$.

It follows from Corollary 2.3 that $b_{n} b_{j}=q^{\bullet} b_{j} b_{n}$ with $\bullet \geq 0$ for all $j \in\{m, \ldots, n\}$. Then, applying $\sigma$ leads to $b_{s(n)} b_{s(j)}=q^{\bullet} b_{s(j)} b_{s(n)}$ with $\bullet \geq 0$ for all $j \in\{m, \ldots, n\}$. Since $s$ is a permutation of $\{m, \ldots, n\}$, this implies that $b_{s(n)} b_{j}=q^{\bullet} b_{j} b_{s(n)}$ with $\bullet \geq 0$ for all $j \in\{m, \ldots, n\}$. Now, if $s(n) \neq n$, then we have $s(n)+1 \in\{m, \ldots, n\}$ and $b_{s(n)} b_{s(n)+1}=q^{-1} b_{s(n)+1} b_{s(n)}$. This is a contradiction and so $s(n)=n$.

We now assume that $m \leq i<n$. It follows from the induction hypothesis that $s$ induces a permutation of $\{m, \ldots, i\}$; so that $s(i) \leq i$. By using a similar argument to that in the previous paragraph, we obtain $s(i)=i$.

Hence, $s(i)=i$ for all $i \in\{m, \ldots, n\}$.

Now let $i \in\{1, \ldots, m-1\}$. Then $b_{i} \in R_{i}$; and so it follows from Proposition 3.2 that $\sigma\left(b_{i}\right) \in R_{\geq i} \backslash R_{>i}$. Hence $b_{s(i)} \in R_{\geq i} \backslash R_{>i}$ so that $b_{s(i)}$ is also a $i \times i$ quantum minor. This implies that either $s(i)=i$ or $s(i)=m+n-i$. Note that similar arguments show 
that either $s(m+n-i)=i$ or $s(m+n-i)=m+n-i$; so $s$ induces a permutation of $\{i, m+n-i\}$. Observe that it follows from Corollary 2.3 that $b_{n} b_{m+n-i}=b_{m+n-i} b_{n}$ and $b_{n} b_{i}=q^{\bullet} b_{i} b_{n}$ with $\bullet>0$. Since we have already proved that $s(n)=n$, applying $\sigma$ leads to $b_{n} b_{s(m+n-i)}=b_{s(m+n-i)} b_{n}$ and $b_{n} b_{s(i)}=q^{\bullet} b_{s(i)} b_{n}$ with $\bullet>0$. This forces $s(i)=i$ and $s(m+n-i)=m+n-i$.

\subsubsection{The case where $n=3 m$.}

Throughout this section, we assume that $n=3 m$. In this case, we are not able to prove directly that the set of all $\mathcal{H}$-invariant height one primes is invariant under every automorphism of $R=\mathcal{O}_{q}\left(M_{m, n}\right)$. However, by using arguments similar to those developed in the proof of Lemma 3.4, one can establish the following weaker result.

Lemma 3.6 Assume that $n=3 m$. Let $\sigma$ be an automorphism of $R$ and $i \in\{1, \ldots, m+$ $n-1\}$.

Then, either

1. there exists $j \in\{1, \ldots, m+n-1\}$ such that $\sigma\left(\left\langle b_{i}\right\rangle\right)=\left\langle b_{j}\right\rangle$,

or,

2. there exist $\lambda \in \mathbb{C}^{*}$ and $\mu \in \mathbb{C}$ such that $\sigma\left(b_{i}\right)=\lambda b_{2 m}+\mu b_{m} b_{3 m}$.

Proof. Note that $v_{2}(m)=v_{2}(n)$, since $n=3 m$. Also, observe that the greatest common divisor $d$ of $m$ and $n$ is equal to $m$ and that, if we set $m=d m^{\prime}$ and $n=d n^{\prime}$, then $m^{\prime}=1$ and $n^{\prime}=3$.

Since $\left\langle b_{i}\right\rangle$ is a height one prime ideal of $R$, its image under $\sigma$ is also a height one prime ideal of $R$. We distinguish between two cases.

If $\left\langle\sigma\left(b_{i}\right)\right\rangle$ is $\mathcal{H}$-invariant, then it follows from Proposition 2.1 that $\sigma\left(\left\langle b_{i}\right\rangle\right)=\left\langle b_{j}\right\rangle$ for some $j$ and so the proof is complete in this case.

Assume now that $\left\langle\sigma\left(b_{i}\right)\right\rangle$ is not $\mathcal{H}$-invariant. In this case, $\left\langle\sigma\left(b_{i}\right)\right\rangle$ is a height one (completely) prime ideal of $R$ which is not $\mathcal{H}$-invariant. Thus it follows from Proposition 2.5 that $\left\langle\sigma\left(b_{i}\right)\right\rangle$ belongs to the $\langle 0\rangle$-stratum of the prime spectrum of $R$; and so, recalling that $d=m$, we deduce from Proposition 2.6 that there exist $\left(r_{1}, \ldots, r_{m}\right) \in \mathbb{N}^{m} \backslash\{0\}$ and scalars $a_{i_{1}, \ldots, i_{m}} \in \mathbb{C}$ such that $\left\langle\sigma\left(b_{i}\right)\right\rangle=\langle u\rangle$ where

$$
u:=\sum_{i_{1}=0}^{r_{1}} \cdots \sum_{i_{m}=0}^{r_{m}} a_{i_{1}, \ldots, i_{m}} \prod_{j=1}^{m}\left[\prod_{\substack{i=0 \\ i \text { even }}}^{3} b_{i m+j}\right]^{i_{j}}\left[\prod_{\substack{i=0 \\ i \text { odd }}}^{3} b_{i m+j}\right]^{r_{j}-i_{j}}
$$


It follows that $u$ is a normal element of $R$, by Proposition 2.6. On the other hand, $\sigma\left(b_{i}\right)$ is normal in $R$, since $b_{i}$ is normal in $R$. . Hence, $u \in \mathbb{C}^{*} \sigma\left(b_{i}\right)$, by Lemma 3.1.

Now $b_{i}$ is a quantum minor of $R=\mathcal{O}_{q}\left(M_{m, n}\right)$; and so $b_{i}$ is a homogeneous element of degree less than or equal to $m$. Thus, Corollary 3.3 implies that

$$
u \notin R_{>m} .
$$

On the other hand, since $\left(r_{1}, \ldots, r_{m}\right) \neq 0$, there exists $k$ such that $r_{k} \geq 1$. We consider three separate cases.

- First, suppose that $k<m$, then

$$
\operatorname{deg}\left(\prod_{j=1}^{m}\left[\prod_{\substack{i=0 \\ i \text { even }}}^{3} b_{i m+j}\right]^{i_{j}}\left[\prod_{\substack{i=0 \\ i \text { odd }}}^{3} b_{i m+j}\right]^{r_{j}-i_{j}}\right) \geq \begin{cases}\operatorname{deg}\left(b_{k} b_{2 m+k}\right) & \text { if } i_{k} \geq 1 \\ \operatorname{deg}\left(b_{m+k} b_{3 m+k}\right) & \text { if } i_{k}=0\end{cases}
$$

Now, $m+k$ and $2 m+k$ are both between $m$ and $n$, since $k<m$ and $n=3 m$. Thus, $b_{m+k}$ and $b_{2 m+k}$ are $m \times m$ quantum minors. Also, $b_{k}$ and $b_{3 m+k}$ are homogeneous of degree greater than or equal to 1 . Thus, in both cases, we get

$$
\operatorname{deg}\left(\prod_{j=1}^{m}\left[\prod_{\substack{i=0 \\ i \text { even }}}^{3} b_{i m+j}\right]^{i_{j}}\left[\prod_{\substack{i=0 \\ i \text { odd }}}^{3} b_{i m+j}\right]^{r_{j}-i_{j}}\right) \geq m+1
$$

Hence $u$ is a linear combination of terms of degree greater than $m$. This implies that $u \in R_{>m}$. This contradicts (5).

- Next, assume that $k=m$ and $r_{m} \geq 2$. In this case, one can prove, by using similar arguments, that $u$ is a linear combination of terms of degree greater than $m$; so this case cannot happen.

- Finally, assume that $k=m$ and $\left(r_{1}, \ldots, r_{m}\right)=(0, \ldots, 0,1)$. In this case, there exist $\lambda^{\prime}, \mu^{\prime} \in \mathbb{C}$ such that

$$
u=\lambda^{\prime} b_{2 m}+\mu^{\prime} b_{m} b_{3 m}
$$

If $\lambda^{\prime}=0$, then once again $u$ is a linear combination of terms of degree greater than $m$, contradicting (5). Hence $\lambda^{\prime} \neq 0$. Since we have already proved that $u \in \mathbb{C}^{*} \sigma\left(b_{i}\right)$, we see that there exist $\lambda \in \mathbb{C}^{*}$ and $\mu \in \mathbb{C}$ such that $\sigma\left(b_{i}\right)=\lambda b_{2 m}+\mu b_{m} b_{3 m}$, as desired.

The following commutation relations can be easily deduced from Corollary 2.3. 
Lemma 3.7 For all $m \leq i \leq 3 m$ :

$$
b_{i}\left(\lambda b_{2 m}+\mu b_{m} b_{3 m}\right)= \begin{cases}q^{\alpha_{i, 2 m}}\left(\lambda b_{2 m}+\mu b_{m} b_{3 m}\right) b_{i} & \text { if } i<2 m \\ q^{-\alpha_{i, 2 m}}\left(\lambda b_{2 m}+\mu b_{m} b_{3 m}\right) b_{i} & \text { if } i>2 m\end{cases}
$$

where $\alpha_{i, 2 m}:=|\{3 m-i+1, \ldots, 4 m-i\} \cap\{m+1, \ldots, 2 m\}|-m$.

We can now obtain the analogous (but slightly weaker) result to Proposition 3.5 in the $n=3 m$ case.

Proposition 3.8 Assume that $n=3 m$ and let $\sigma$ be an automorphism of $R$. Then, for all $i \in\{1, \ldots, 4 m-1\}, i \neq 2 m$, there exists $\lambda_{i} \in \mathbb{C}^{*}$ such that $\sigma\left(b_{i}\right)=\lambda_{i} b_{i}$.

Proof. Let $i \in\{1, \ldots, 4 m-1\}$ and assume that there exists $j \in\{1, \ldots, 4 m-1\}$ such that $\sigma\left(\left\langle b_{i}\right\rangle\right)=\left\langle b_{j}\right\rangle$. Then, it follows from Lemma 3.1 that there exists $\lambda_{j} \in \mathbb{C}^{*}$ such that $\sigma\left(b_{i}\right)=\lambda_{i} b_{j}$, since $\sigma\left(b_{i}\right)$ and $b_{j}$ are normal.

Thus, we deduce from Lemma [3.6] that, for all $i \in\{1, \ldots, 4 m-1\}$, either there exist $j \in\{1, \ldots, 4 m-1\}$ and $\lambda_{i} \in \mathbb{C}^{*}$ such that $\sigma\left(b_{i}\right)=\lambda_{i} b_{j}$, or there exist $\lambda_{i}, \mu_{i} \in \mathbb{C}$ with $\lambda_{i} \neq 0$ such that $\sigma\left(b_{i}\right)=\lambda_{i} b_{2 m}+\mu_{i} b_{m} b_{3 m}$.

We distinguish between two cases.

- If, for each $i \in\{1, \ldots, 4 m-1\}$, there exist $j \in\{1, \ldots, 4 m-1\}$ and $\lambda_{i} \in \mathbb{C}^{*}$ such that $\sigma\left(b_{i}\right)=\lambda_{i} b_{j}$, then, by using similar arguments to those in the proof of Proposition [3.5, we show that $\sigma\left(b_{i}\right)=\lambda_{i} b_{i}$ for all $i \in\{1, \ldots, 4 m-1\}$.

- Now, assume that there exist $k \in\{1, \ldots, 4 m-1\}$, and $\lambda_{k}, \mu_{k} \in \mathbb{C}$ with $\lambda_{k} \neq 0$, such that $\sigma\left(b_{k}\right)=\lambda_{k} b_{2 m}+\mu_{k} b_{m} b_{3 m}$.

• First, we show that $k=2 m$.

Observe that, for all $i \in\{1, \ldots, m-1\} \cup\{3 m+1, \ldots, 4 m-1\}$, the quantum minor $b_{i}$ is a homogeneous element of degree less than $m$. Hence, $\sigma\left(b_{i}\right) \notin R_{\geq m}$, by Corollary [3.3. Since $\sigma\left(b_{k}\right)=\lambda_{k} b_{2 m}+\mu_{k} b_{m} b_{3 m} \in R_{\geq m}$, this shows that $i \neq k$. Thus, $k \in\{m, \ldots, 3 m\}$.

Next, let $i \in\{m, \ldots, 3 m\}$, so that $b_{i}$ is a $m \times m$ quantum minor. Then $b_{i} \in R_{m}$ and it follows from Corollary 3.3 that $\sigma\left(b_{i}\right) \in R_{\geq m}$. Hence, either

$$
\text { there exist } j \in\{m, \ldots, 3 m\} \text { and } \lambda_{i} \in \mathbb{C}^{*} \text { such that } \sigma\left(b_{i}\right)=\lambda_{i} b_{j} \text {, }
$$

or

there exist $\lambda_{i}, \mu_{i} \in \mathbb{C}$ with $\lambda_{i} \neq 0$ such that $\sigma\left(b_{i}\right)=\lambda_{i} b_{2 m}+\mu_{i} b_{m} b_{3 m}$. 
We now prove that (77) can not happen, for all $i \in\{2 m+1, \ldots, 3 m\}$. Indeed, assume that there exists $i \in\{2 m+1, \ldots, 3 m\}$ such that $\sigma\left(b_{i}\right)=\lambda_{i} b_{2 m}+\mu_{i} b_{m} b_{3 m}$. Note that $b_{i} b_{j}=q^{\bullet} b_{j} b_{i}$ with $\bullet>0$, for all $m \leq j<i$, by Corollary 2.3 ,

Hence, by applying $\sigma$, we obtain:

$$
\left(\lambda_{i} b_{2 m}+\mu_{i} b_{m} b_{3 m}\right) \sigma\left(b_{j}\right)=q^{\bullet} \sigma\left(b_{j}\right)\left(\lambda_{i} b_{2 m}+\mu_{i} b_{m} b_{3 m}\right)
$$

with $\bullet>0$ for all $m \leq j<i$. This implies that $\sigma\left(b_{j}\right)$ can not be equal to $\lambda b_{2 m}+\mu b_{m} b_{3 m}$ with $\lambda, \mu \in \mathbb{C}$, since $\lambda b_{2 m}+\mu b_{m} b_{3 m}$ commutes with $\lambda_{i} b_{2 m}+\mu_{i} b_{m} b_{3 m}$ by Lemma 3.7 Hence we deduce from (66) and (77) that, for each $j$ such that $m \leq j<i$, there exist $l \in\{m, \ldots, 3 m-1\}$ and $\lambda_{j} \in \mathbb{C}^{*}$ such that $\sigma\left(b_{j}\right)=\lambda_{j} b_{l}$. Moreover, we deduce from (8) and Lemma 3.7 that we must have $l \in\{m, \ldots, 2 m-1\}$. Thus, since $i>2 m$, there exist $j \neq j^{\prime}$ with $m \leq j, j^{\prime}<i$ with $\sigma\left(b_{j}\right) \in \mathbb{C}^{*} \sigma\left(b_{j^{\prime}}\right)$. This is impossible since $\sigma$ is an automorphism and $\left\langle b_{j}\right\rangle \neq\left\langle b_{j^{\prime}}\right\rangle$, by Proposition 2.1]

Hence, for all $i \in\{2 m+1, \ldots, 3 m\}$, there exist $j \in\{m, \ldots, 3 m\}$ and $\lambda_{i} \in \mathbb{C}^{*}$ such that $\sigma\left(b_{i}\right)=\lambda_{i} b_{j}$. In other words, $k \neq i$ for all $i \in\{2 m+1, \ldots, 3 m\}$.

A similar argument shows that, for all $i \in\{m, \ldots, 2 m-1\}$, there exist $j \in\{m, \ldots, 3 m\}$ and $\lambda_{i} \in \mathbb{C}^{*}$ such that $\sigma\left(b_{i}\right)=\lambda_{i} b_{j}$, and so $k \neq i$ for all $i \in\{m, \ldots, 2 m-1\}$.

In conclusion, the only possibility is $k=2 m$. Hence, we have already proved that:

1. There exist $\lambda, \mu \in \mathbb{C}$, with $\lambda \neq 0$, such that $\sigma\left(b_{2 m}\right)=\lambda b_{2 m}+\mu b_{m} b_{3 m}$.

2. For all $i \in\{m, \ldots, 3 m\}$ with $i \neq 2 m$, there exist $j \in\{m, \ldots, 3 m\}$ and $\lambda_{i} \in \mathbb{C}^{*}$ such that $\sigma\left(b_{i}\right)=\lambda_{i} b_{j}$.

3. For all $i \in\{1, \ldots, m-1\} \cup\{3 m+1, \ldots, 4 m-1\}$, there exist $j \in\{1, \ldots, 4 m-1\}$ and $\lambda_{i} \in \mathbb{C}^{*}$ such that $\sigma\left(b_{i}\right)=\lambda_{i} b_{j}$.

$\bullet$ We prove by induction that $\sigma\left(b_{i}\right)=\lambda_{i} b_{i}$, for all $i \in\{2 m+1, \ldots, 3 m\}$.

First, we know that there exist $\lambda_{2 m+1} \in \mathbb{C}^{*}$ and $j \in\{m, \ldots, 3 m\}$ such that $\sigma\left(b_{2 m+1}\right)=$ $\lambda_{2 m+1} b_{j}$. It follows from Corollary 2.3 that $b_{2 m} b_{2 m+1}=q b_{2 m+1} b_{2 m}$. Hence, applying $\sigma$ yields:

$$
\left(\lambda b_{2 m}+\mu b_{m} b_{3 m}\right) b_{j}=q b_{j}\left(\lambda b_{2 m}+\mu b_{m} b_{3 m}\right) .
$$

In view of Lemma 3.7, this forces $j=2 m+1$, as desired.

Next, let $i \in\{2 m+2, \ldots, 3 m\}$. It follows from the previous study that there exist $j \in\{m, \ldots, 3 m\}$ and $\lambda_{i} \in \mathbb{C}^{*}$ such that $\sigma\left(b_{i}\right)=\lambda_{i} b_{j}$. Moreover we deduce from the 
induction hypothesis that $\sigma\left(b_{i-1}\right)=\lambda_{i-1} b_{i-1}$. Now, Corollary 2.3 shows that $b_{i} b_{i-1}=$ $q b_{i-1} b_{i}$. Applying $\sigma$ yields

$$
b_{j} b_{i-1}=q b_{i-1} b_{j} .
$$

In view of Corollary 2.3, this implies that $j=i$, as desired.

Hence, $\sigma\left(b_{i}\right)=\lambda_{i} b_{i}$, for all $i \in\{2 m+1, \ldots, 3 m\}$. A similar argument shows that $\sigma\left(b_{i}\right)=\lambda_{i} b_{i}$, for all $i \in\{m, \ldots, 2 m-1\}$.

-• Now, let $i \in\{1, \ldots, m-1\}$. Then $b_{i} \in R_{i}$; and so it follows from Proposition 3.2 that $\sigma\left(b_{i}\right) \in R_{\geq i} \backslash R_{>i}$. Now, there exist $j \in\{1, \ldots, 4 m-1\}$ and $\lambda_{i} \in \mathbb{C}^{*}$ such that $\sigma\left(b_{i}\right)=\lambda_{i} b_{j}$ because of the previous study. Hence we have $b_{j} \in R_{\geq i} \backslash R_{>i}$, so that $b_{j}$ is also a $i \times i$ quantum minor. This implies that either $j=i$ or $j=4 m-i$. Now, it follows from Corollary 2.3 that $b_{3 m} b_{i}=q^{\bullet} b_{i} b_{3 m}$ with $\bullet>0$. Since we have already proved that $\sigma\left(b_{3 m}\right)=\lambda_{3 m} b_{3 m}$, composing by $\sigma$ leads to $b_{3 m} b_{j}=q^{\bullet} b_{j} b_{3 m}$ with $\bullet>0$. On the other hand, it follows Corollary 2.3 that $b_{3 m} b_{4 m-i}=b_{4 m-i} b_{3 m}$. So $j$ can not be equal to $4 m-i$. Hence $j=i$ and so $\sigma\left(b_{i}\right)=\lambda_{i} b_{i}$, as desired.

A similar argument shows that there exist $\lambda_{i} \in \mathbb{C}^{*}$ such that $\sigma\left(b_{i}\right)=\lambda_{i} b_{i}$, for all $i \in\{3 m+1, \ldots, 4 m-1\}$.

\subsection{The automorphism group of non-square quantum matrices.}

Theorem 3.9 Assume that $m<n$ and $(m, n) \neq(1,3)$. Let $\sigma$ be an automorphism of $\mathcal{O}_{q}\left(M_{m, n}\right)$. Then there exist $\mu_{i, \alpha} \in \mathbb{C}^{*}$ such that $\sigma\left(Y_{i, \alpha}\right)=\mu_{i, \alpha} Y_{i, \alpha}$, for all $(i, \alpha) \in \llbracket 1, m \rrbracket \times$ $\llbracket 1, n \rrbracket$.

Proof. We proceed by induction on $n$. The case $n=2$ easily follows from Proposition 3.5. So we assume that $n \geq 3$. If $m=1$, then $n>3$, and so once again the result easily follows from Proposition 3.5. So we assume that $m \geq 2$. We need to see that $\sigma$ acts on each generator $Y_{i, \alpha}$ by multiplication by a scalar. We do this by using preferred basis arguments. We use the language and notation of [7].

Note that, because of [7, Proposition 5.3], the quantum minor $b_{n}=[1 \ldots m \mid 1 \ldots m]$ commutes with $Y_{i, \alpha}$, for $1 \leq i, \alpha \leq m$, and that $b_{n}[R \mid C]=q^{\bullet}[R \mid C] b_{n}$ with $\bullet \geq 0$ for all other quantum minors $[R \mid C]$ of $\mathcal{O}_{q}\left(M_{m, n}\right)$. Thus, $b_{n} q^{\bullet}$-commutes with each monomial in the preferred basis where $\bullet$ is $\geq 0$, and [7, Proposition 5.3] shows that $\bullet$ is equal to zero if and only if the only quantum minors that occur in the monomial are those of $\left(Y_{i, \alpha}\right)_{i, \alpha \in\{1, \ldots, m\}}$. Observe further that, if $b_{n}=[1 \ldots m \mid 1 \ldots m]$ commutes with an element 
$y \in \mathcal{O}_{q}\left(M_{m, n}\right)$, then it must commute with each of the monomials in the expression for $y$ in the preferred basis.

Now, let $\sigma$ be an automorphism of $\mathcal{O}_{q}\left(M_{m, n}\right)$. We will show first that $\sigma\left(Y_{i, \alpha}\right)=\mu_{i, \alpha} Y_{i, \alpha}$ for all $1 \leq i, \alpha \leq m$.

Set $y_{i, \alpha}:=\sigma\left(Y_{i, \alpha}\right)$. Since $Y_{i, \alpha}$ commutes with $b_{n}$ and since $\sigma$ acts on $b_{n}$ by multiplication by a scalar (see Proposition 3.5 if $n \neq 3 m$ or Proposition 3.8 if $n=3 m$ ), then $y_{i, \alpha}$ must commute with $b_{n}$. Thus, any monomial $z$ in the expression for $y_{i, \alpha}$ in the preferred basis must also commute with $b_{n}$. This means that the only quantum minors that can occur in $z$ are those of $\left(Y_{i, \alpha}\right)_{i, \alpha \in\{1, \ldots, m\}}$. In particular, $\sigma\left(Y_{i, \alpha}\right)$ belongs to the subalgebra $R_{m, m}$ of $R=\mathcal{O}_{q}\left(M_{m, n}\right)$ generated by $Y_{i, \alpha}, 1 \leq i, \alpha \leq m$ (which is a copy of $O_{q}\left(M_{m, m}\right)$ ). Hence $\sigma$ induces an automorphism of $R_{m, m}$.

Moreover, $\sigma$ acts on the quantum minors $b_{n+i}=[i+1, \ldots, m \mid 1, \ldots, m-i]$ by multiplication by scalars, by Proposition 3.5 (if $n \neq 3 m$ ) or Proposition 3.8 (if $n=3 m$ ). In particular, there exists $\mu_{m, 1} \in \mathbb{C}^{*}$ such that $\sigma\left(Y_{m, 1}\right)=\mu_{m, 1} Y_{m, 1}$. Let $j \in\{1, \ldots, m-1\}$. Then $Y_{j, 1} Y_{m, 1}=q Y_{m, 1} Y_{j, 1}$; so that $\sigma\left(Y_{j, 1}\right) Y_{m, 1}=q Y_{m, 1} \sigma\left(Y_{j, 1}\right)$. Write $\sigma\left(Y_{j, 1}\right)$ in the PBW basis of $R_{m, m}$ :

$$
\sigma\left(Y_{j, 1}\right)=\sum_{\underline{\gamma} \in \Gamma} c_{\underline{\gamma}} Y_{1,1}^{\gamma_{1,1}} Y_{1,2}^{\gamma_{1,2}} \ldots Y_{m, m}^{\gamma_{m, m}},
$$

where $\Gamma$ is a finite subset of $\mathbb{N}^{m^{2}}$. Hence $\sigma\left(Y_{j, 1}\right) Y_{m, 1}=q Y_{m, 1} \sigma\left(Y_{j, 1}\right)$ implies that, for all $\underline{\gamma} \in \Gamma$ such that $c_{\underline{\gamma}} \neq 0$, we have $\sum_{i=1}^{m-1} \gamma_{i, 1}-\sum_{i=2}^{m} \gamma_{m, i}=1$. Thus, for all $\underline{\gamma} \in \Gamma$ such that $c_{\underline{\gamma}} \neq 0$, there exists $i \in\{1, \ldots, m-1\}$ with $\gamma_{i, 1} \neq 0$. Denote by $J$, the prime ideal of $R$ generated by the $Y_{i, 1}$, with $i \in\{1, \ldots, m\}$, and, similarly, denote by $J^{\prime}$ the prime ideal of $R_{m, m}$ generated by the $Y_{i, 1}$, with $i \in\{1, \ldots, m\}$. Observe that $J=Y_{1,1} R+\cdots+Y_{m, 1} R$ and $J^{\prime}=Y_{1,1} R_{m, m}+\cdots+Y_{m, 1} R_{m, m}$. We have just proved that $\sigma\left(Y_{j, 1}\right) \in J^{\prime}$ for all $j \in\{1, \ldots, m\}$. Hence $\sigma\left(J^{\prime}\right)=J^{\prime}$ and $\sigma(J)=J$.

Thus, $\sigma$ induces an automorphism of $R_{m, m} / J^{\prime} \simeq O_{q}\left(M_{m, m-1}\right)$. Now, because of the induction hypothesis (and the isomorphism $O_{q}\left(M_{m, m-1}\right) \simeq O_{q}\left(M_{m-1, m}\right)$ ), we obtain $\sigma\left(Y_{i, \alpha}\right)=\mu_{i, \alpha} Y_{i, \alpha}+\sum_{\underline{\gamma} \in \Gamma} c_{\underline{\gamma}} Y_{1,1}^{\gamma_{1,1}} Y_{1,2}^{\gamma_{1,2}} \ldots Y_{m, m}^{\gamma_{m, m}}$ with $\mu_{i, \alpha} \neq 0$ and at least one of $\gamma_{k, 1}>0$ for each nonzero term of the sum. (Observe that we can apply the inductive hypothesis since $(m-1, m) \neq(1,3)$.)

On the other hand, it follows from Proposition 3.5 or Proposition 3.8 that 
$\sigma\left(b_{n-1}\right)=\lambda b_{n-1}$ with $\lambda \in \mathbb{C}^{*}$. Moreover, recalling that $b_{n-1}=[1 \ldots m \mid 2 \ldots m+1]$, we deduce from a transposed version of [7. Lemma 5.1] that, for all $1 \leq i, \alpha \leq m$, we have

$$
b_{n-1} Y_{i, \alpha}=q^{\bullet} Y_{i, \alpha} b_{n-1}
$$

with $\bullet=-1$ if $i=1$ and 0 otherwise. Let $1 \leq i, \alpha \leq m$ with $\alpha \neq 1$. Thus we must have $b_{n-1} \sigma\left(Y_{i, \alpha}\right)=\sigma\left(Y_{i, \alpha}\right) b_{n-1}$. Since $\sigma\left(Y_{i, \alpha}\right)=\mu_{i, \alpha} Y_{i, \alpha}+\sum_{\gamma \in \Gamma} c_{\gamma} Y_{1,1}^{\gamma_{1,1}} Y_{1,2}^{\gamma_{1,2}} \ldots Y_{m, m}^{\gamma_{m, m}}$ with at least one of $\gamma_{k, 1} \neq 0$ for each nonzero term of the sum, this implies that the sum is empty and so $\sigma\left(Y_{i, \alpha}\right)=\mu_{i, \alpha} Y_{i, \alpha}$ for all $1 \leq i, \alpha \leq m$ with $\alpha \neq 1$.

Now $\sigma$ induces an automorphism of $R_{m, m}$ that acts on the indeterminates $Y_{i, \alpha}(1 \leq i \leq$ $m$ and $1<\alpha \leq m$ ) by multiplication by scalars. Moreover, it follows from Proposition 3.5 or Proposition 3.8 that $\sigma$ also acts on the $b_{i}=[i-n+1, \ldots, m \mid 1, \ldots, m+n-i]$ $(n \leq i \leq m+n-1)$ by multiplication by scalars. This forces $\sigma\left(Y_{i, 1}\right)=\mu_{i, 1} Y_{i, 1}$ for all $1 \leq i \leq m$.

This establishes the following claim:

Claim 3.10 For all $(i, \alpha) \in \llbracket 1, m \rrbracket^{2}$, there exists $\mu_{i, \alpha} \in \mathbb{C}^{*}$ such that $\sigma\left(Y_{i, \alpha}\right)=\mu_{i, \alpha} Y_{i, \alpha}$.

It remains to consider the case that $\alpha>m$. Let us now distinguish between two cases.

If $n=m+1$, then $\sigma$ is an automorphism of $R=O_{q}\left(M_{m, m+1}\right)$ that acts on the indeterminates $Y_{i, \alpha}(1 \leq i, \alpha \leq m)$ by multiplication by scalars. Further, because of Proposition 3.5 or Proposition 3.8, $\sigma$ also acts on the quantum minors $b_{i}=[1, \ldots, i \mid n-i+1, \ldots, n]$ $(1 \leq i \leq m)$ by multiplication by scalars. This forces $\sigma\left(Y_{i, m+1}\right)=\mu_{i, m+1} Y_{i, m+1}$ for all $1 \leq i \leq m$. This finishes the proof of Theorem 3.9 in the case where $n=m+1$.

Now, assume that $m+1<n$. Recall that $J$ denotes the two-sided ideal generated by the $Y_{k, 1}, 1 \leq k \leq m$. Note that $J=Y_{1,1} R+\cdots+Y_{m, 1} R$. It is well-known that the monomials

$$
Y_{1,1}^{\gamma_{1,1}} Y_{2,1}^{\gamma_{2,1}} \ldots Y_{m, 1}^{\gamma_{m, 1}} \ldots Y_{1, n}^{\gamma_{1, n}} \ldots Y_{m, n}^{\gamma_{m, n}}
$$

form a PBW basis of $R$. Observe that $x=\sum_{\underline{\gamma} \in \Gamma} c_{\underline{\gamma}} Y_{1,1}^{\gamma_{1,1}} \ldots Y_{m, 1}^{\gamma_{m, 1}} \ldots Y_{1, n}^{\gamma_{1, n}} \ldots Y_{m, n}^{\gamma_{m, n}}$ belongs to $J$ if and only if at least one of the $\gamma_{k, 1} \geq 1$ for all $\gamma \in \Gamma$ such that $c_{\underline{\gamma}} \neq 0$.

It follows from Claim 3.10 that $\sigma(J)=J$. Hence $\sigma$ induces an automorphism of $R / J \simeq \mathcal{O}_{q}\left(M_{m, n-1}\right)$. Since $m<n-1$ and $m \geq 2$, it follows from the inductive hypothesis that, for all $(i, \alpha)$ with $m+1 \leq \alpha \leq n$, we can write

$$
\sigma\left(Y_{i, \alpha}\right)=\mu_{i, \alpha} Y_{i, \alpha}+\sum_{\underline{\gamma} \in \Gamma} c_{\underline{\gamma}} Y_{1,1}^{\gamma_{1,1}} \ldots Y_{m, 1}^{\gamma_{m}, 1} \ldots Y_{1, n}^{\gamma_{1, n}} \ldots Y_{m, n}^{\gamma_{m, n}}
$$


where $\Gamma$ is a finite subset of $\mathbb{N}^{m n}$ and at least one of the $\gamma_{k, 1} \geq 1$ for all $\gamma \in \Gamma$ such that $c_{\underline{\gamma}} \neq 0$.

Let $K$ the two-sided ideal generated by the $Y_{k, 2}, 1 \leq k \leq m$. Note that an element

$$
x=\sum_{\underline{\gamma} \in \Gamma^{\prime}} c_{\gamma} Y_{1,1}^{\gamma_{1,1}} \ldots Y_{m, 1}^{\gamma_{m, 1}} \ldots Y_{1, n}^{\gamma_{1, n}} \ldots Y_{m, n}^{\gamma_{m, n}} \in R
$$

belongs to $K$ if and only if at least one of the $\gamma_{k, 2} \geq 1$ for all $\gamma \in \Gamma^{\prime}$ such that $c_{\underline{\gamma}} \neq 0$.

It follows from Claim 3.10 that $\sigma(K)=K$. Hence $\sigma$ induces an automorphism of $R / K \simeq \mathcal{O}_{q}\left(M_{m, n-1}\right)$. Since $m<n-1$ and $m \geq 2$, the inductive hypothesis applies and, for all $(i, \alpha)$ with $m+1 \leq \alpha \leq n$, we can write

$$
\sigma\left(Y_{i, \alpha}\right)=\mu_{i, \alpha}^{\prime} Y_{i, \alpha}+\sum_{\underline{\gamma} \in \Gamma^{\prime}} c_{\underline{\gamma}} Y_{1,1}^{\gamma_{1,1}} \ldots Y_{m, 1}^{\gamma_{m, 1}} \ldots Y_{1, n}^{\gamma_{1, n}} \ldots Y_{m, n}^{\gamma_{m, n}}
$$

where $\Gamma^{\prime}$ is a finite subset of $\mathbb{N}^{m n}$ and at least one of the $\gamma_{k, 2} \geq 1$ for all $\gamma \in \Gamma^{\prime}$ such that $c_{\underline{\gamma}} \neq 0$.

Let $(i, \alpha)$ with $m+1 \leq \alpha \leq n$. It remains to prove that $\sigma$ acts on $Y_{i, \alpha}$ by multiplication by a scalar. First, identifying the two expressions (9) and (10) of $\sigma\left(Y_{i, \alpha}\right)$ in the PBW basis of $R$ leads to:

$$
\sigma\left(Y_{i, \alpha}\right)=\mu_{i, \alpha} Y_{i, \alpha}+\sum_{\underline{\gamma} \in \Gamma} c_{\underline{\gamma}} Y_{1,1}^{\gamma_{1,1}} \ldots Y_{m, 1}^{\gamma_{m, 1}} \ldots Y_{1, n}^{\gamma_{1, n}} \ldots Y_{m, n}^{\gamma_{m, n}}
$$

where $\Gamma$ is a finite subset of $\mathbb{N}^{m n}$ such that at least one of the $\gamma_{k, 1} \geq 1$ and at least one of the $\gamma_{l, 2} \geq 1$ for all $\gamma \in \Gamma$ such that $c_{\underline{\gamma}} \neq 0$.

By Proposition 3.5 (if $n \neq 3 m$ ) or Proposition 3.8 (if $n=3 m$ ), the automorphism $\sigma$ acts on $b_{m}=[1 \ldots m \mid n-m+1 \ldots n]$ by multiplication by a scalar. Further, it follows from a transposed version of [7, Lemma 5.1] that

$$
b_{m} Y_{i, \alpha}= \begin{cases}q^{-1} Y_{i, \alpha} b_{m} & \text { if } \alpha \leq n-m \\ Y_{i, \alpha} b_{m} & \text { if } \alpha \geq n-m+1\end{cases}
$$

Hence

$$
b_{m} \sigma\left(Y_{i, \alpha}\right)= \begin{cases}q^{-1} \sigma\left(Y_{i, \alpha}\right) b_{m} & \text { if } \alpha \leq n-m \\ \sigma\left(Y_{i, \alpha}\right) b_{m} & \text { if } \alpha \geq n-m+1\end{cases}
$$

Thus

$$
\sum_{\underline{\gamma} \in \Gamma} c_{\underline{\gamma}} q^{-\gamma_{1,1}-\cdots-\gamma_{m, 1}-\cdots-\gamma_{1, n-m}-\cdots-\gamma_{m, n-m}} Y_{1,1}^{\gamma_{1,1}} \ldots Y_{m, 1}^{\gamma_{m, 1}} \ldots Y_{1, n}^{\gamma_{1, n}} \ldots Y_{m, n}^{\gamma_{m, n}}
$$




$$
=q^{\bullet} \sum_{\underline{\gamma} \in \Gamma} c_{\underline{\gamma}} Y_{1,1}^{\gamma_{1,1}} \ldots Y_{m, 1}^{\gamma_{m, 1}} \ldots Y_{1, n}^{\gamma_{1, n}} \ldots Y_{m, n}^{\gamma_{m, n}},
$$

where $q^{\bullet}=-1$ if $\alpha \leq n-m$, and $q^{\bullet}=0$ otherwise.

Consequently,

$$
\gamma_{1,1}+\cdots+\gamma_{m, 1}+\cdots+\gamma_{1, n-m}+\cdots+\gamma_{m, n-m}= \begin{cases}1 & \text { if } \alpha \leq n-m \\ 0 & \text { if } \alpha \geq n-m+1\end{cases}
$$

for all $\underline{\gamma} \in \Gamma$ such that $c_{\underline{\gamma}} \neq 0$, since $q$ is not a root of unity.

On the other hand, recall that

$$
\sigma\left(Y_{i, \alpha}\right)=\mu_{i, \alpha} Y_{i, \alpha}+\sum_{\underline{\gamma} \in \Gamma} c_{\underline{\gamma}} Y_{1,1}^{\gamma_{1,1}} \ldots Y_{m, 1}^{\gamma_{m, 1}} \ldots Y_{1, n}^{\gamma_{1, n}} \ldots Y_{m, n}^{\gamma_{m, n}}
$$

where, for each nonzero term of the sum, at least one of the $\gamma_{k, 1}$ is a positive integer and at least one of the $\gamma_{l, 2}$ is a positive integer. Hence, $\gamma_{1,1}+\cdots+\gamma_{m, 1}+\cdots+\gamma_{1, n-m}+\cdots+\gamma_{m, n-m} \geq$ 2 for all $\underline{\gamma} \in \Gamma$ with $c_{\underline{\gamma}} \neq 0$, since $n-m \geq 2$. This contradicts (12). Thus $\Gamma$ must be empty for all $(i, \alpha)$ with $\alpha \geq m$ and so $\sigma\left(Y_{i, \alpha}\right)=\mu_{i, \alpha} Y_{i, \alpha}$ for all $\alpha>m$. This finishes the proof.

Corollary 3.11 Assume that $m<n$ and $(m, n) \neq(1,3)$. Let $\sigma$ be an automorphism of $\mathcal{O}_{q}\left(M_{m, n}\right)$. Then there exist unique nonzero complex numbers $h_{1}, \ldots, h_{m}, h_{1}^{\prime}, \ldots, h_{n-1}^{\prime}$ such that $\sigma\left(Y_{i, \alpha}\right)=h_{i} h_{\alpha}^{\prime} Y_{i, \alpha}$ (with the convention $h_{n}^{\prime}=1$ ).

Thus, $\operatorname{Aut}\left(\mathcal{O}_{q}\left(M_{m, n}\right)\right)$ is isomorphic to the torus $\left(\mathbb{C}^{*}\right)^{m+n-1}$.

Proof. Let $\sigma$ be an automorphism of $\mathcal{O}_{q}\left(M_{m, n}\right)$. By Theorem [3.9, there exists a family $\left(\mu_{i, \alpha}\right)_{(i, \alpha) \in[1, m] \times[1, n]}$ of elements of $\mathbb{C}^{*}$ such that $\sigma\left(Y_{i, \alpha}\right)=\mu_{i, \alpha} Y_{i, \alpha}$ for all $(i, \alpha) \in \llbracket 1, m \rrbracket \times$ $\llbracket 1, n \rrbracket$. Recall that, if $(i, \alpha),(j, \beta) \in \llbracket 1, m \rrbracket \times \llbracket 1, n \rrbracket$ with $i<j$ and $\alpha<\beta$, then $Y_{j, \beta} Y_{i, \alpha}=$ $Y_{i, \alpha} Y_{j, \beta}-\left(q-q^{-1}\right) Y_{i, \beta} Y_{j, \alpha}$. Hence, since $\sigma$ is an automorphism, we must have $\mu_{i, \alpha} \mu_{j, \beta}=$ $\mu_{i, \beta} \mu_{j, \alpha}$ for all $(i, \alpha),(j, \beta) \in \llbracket 1, m \rrbracket \times \llbracket 1, n \rrbracket$ with $i<j$ and $\alpha<\beta$. In other words, the matrix $\left(\mu_{i, \alpha}\right)_{(i, \alpha) \in[1, m \rrbracket \times \llbracket 1, n]}$ has rank 1 . Hence, there exist unique nonzero complex numbers $h_{1}, \ldots, h_{m}, h_{1}^{\prime}, \ldots, h_{n-1}^{\prime}, h_{n}^{\prime}=1$ such that $\mu_{i, \alpha}=h_{i} h_{\alpha}^{\prime}$ for all $i, \alpha$, as desired.

Note that, in the exceptional case where $(m, n)=(1,3)$, the automorphism group of $\mathcal{O}_{q}\left(M_{1,3}\right)$ has been computed by Alev and Chamarie, [1, Théorème 1.4.6]; in this case, the group is not isomorphic to the torus $\left(\mathbb{C}^{*}\right)^{3}$, since the second case of Lemma 3.6 does arise. Indeed, Alev and Chamarie show that any automorphism of $\mathcal{O}_{q}\left(M_{1,3}\right)$ is of the form

$$
\sigma\left(Y_{11}\right)=\mu_{1} Y_{11}, \quad \sigma\left(Y_{12}\right)=\mu_{2} Y_{12}+\lambda Y_{11} Y_{13}, \quad \sigma\left(Y_{13}\right)=\mu_{3} Y_{13}
$$


where $\mu_{i} \neq 0$ and $\lambda$ are complex numbers; so that the automorphism group is isomorphic to the semidirect product $\mathbb{C} \times\left(\mathbb{C}^{*}\right)^{3}$. This result can easily be obtained from our analysis.

Acknowledgments. We thank Jacques Alev, Ken Goodearl and Laurent Rigal for helpful conversations and comments.

\section{References}

[1] J Alev and M Chamarie, Dérivations et automorphismes de quelques algèbres quantiques, Comm Algebra 20 (6) (1992), 1787-1802

[2] J Alev and F Dumas, Rigidité des plongements des quotients primitifs minimaux de $U_{q}(s l(2))$ dans l'algèbre quantique de Weyl-Hayashi, Nagoya Math J 143 (1996), 119-146

[3] K A Brown and K R Goodearl, Lectures on algebraic quantum groups. Advanced Courses in Mathematics-CRM Barcelona. Birkhäuser Verlag, Basel, 2002.

[4] G Cauchon, Effacement des dérivations et spectres premiers des algèbres quantiques, J Algebra 260 (2003), 476-518.

[5] G Cauchon, Spectre premier de $\mathcal{O}_{q}\left(M_{n}(k)\right)$ image canonique et séparation normale, J Algebra 260 (2003), 519-569

[6] J Gómez-Torrecillas and L El Kaoutit, The group of automorphisms of the coordinate ring of quantum symplectic space, Beiträge Algebra Geom 43 (2002), no. 2, 597-601

[7] K R Goodearl and T H Lenagan, Quantum determinantal ideals, Duke Math J 103 (2000), 165-190.

[8] K R Goodearl and E S Letzter, Prime and primitive spectra of multiparameter quantum affine spaces, in Trends in Ring Theory (Miskolc, 1996) (V Dlab and L Marki, eds), Canad Math Soc Conf Proc Series 22 (1998), 39-58.

[9] K R Goodearl and E S Letzter, The Dixmier-Moeglin equivalence in quantum coordinate rings and quantized Weyl algebras, Trans Amer Math Soc 352 (2000), $1381-1403$ 
[10] K R Goodearl and E S Letzter, Prime factor algebras of the coordinate ring of quantum matrices, Proc Amer Math Soc 121 (1994), 1017-1025

[11] K R Goodearl and R B Warfield, An introduction to noncommutative Noetherian rings. Second edition. London Mathematical Society Student Texts, 61. Cambridge University Press, Cambridge, 2004

[12] H W E Jung, Über ganze birationale Transformationen der Ebene, J Reine Angew Math 184, (1942), 161-174

[13] S Launois, Primitive ideals and automorphism group of $U_{q}^{+}\left(B_{2}\right)$, posted at math.RA/0412358

[14] S Launois, T H Lenagan and L Rigal, Quantum unique factorisation domains, posted at math.QA/0501545

[15] J C McConnell and J C Robson, Noncommutative Noetherian Rings, WileyInterscience, Chichester, 1987

[16] V G Mosin and A N Panov, Division rings of quotients and central elements of multiparameter quantizations, Mat. Sb. 187 (1996), no. 6, 53-72; translation in Sb. Math. 187 (1996), no. 6, 835-855.

[17] B Parshall and J Wang, Quantum linear groups, Mem Amer Math Soc 89 (1991), no. 439

[18] L Rigal, Spectre de l'algbre de Weyl quantique, Beiträge Algebra Geom 37 (1996), no. $1,119-148$

[19] I P Shestakov and U U Umirbaev, The tame and the wild automorphisms of polynomial rings in three variables, J Amer Math Soc 17 (2004), no. 1, 197-227 
S Launois:

School of Mathematics, University of Edinburgh,

James Clerk Maxwell Building, King's Buildings, Mayfield Road, Edinburgh EH9 3JZ, Scotland

E-mail: stephane.launois@ed.ac.uk

T H Lenagan:

School of Mathematics, University of Edinburgh,

James Clerk Maxwell Building, King's Buildings, Mayfield Road, Edinburgh EH9 3JZ, Scotland

E-mail: tom@maths.ed.ac.uk 